\title{
Transforming Triplet Vinylnitrene into Triplet Alkylnitrene at Cryogenic Temperatures
}

Dylan J. Shields, ${ }^{\dagger \times S u j a n ~ K . ~ S a r k a r, ~}{ }^{\dagger} \times$ H. Dushanee M. Sriyarathne, ${ }^{\dagger}$ Jocelyn R. Brown, ${ }^{\dagger}$ Curt Wentrup, $\ddagger$ Manabu Abe, $§$ and Anna D. Gudmundsdottir ${ }^{*} \dagger$

†Department of Chemistry, University of Cincinnati, Cincinnati, OH 45220-0172, United States

₹School of Chemistry and Molecular Bioscience, University of Queensland, Brisbane, QLD 4072, Australia

\$Department of Chemistry, Graduate School of Science, Hiroshima University, 1-3-1 Kagamiyama, HigashiHiroshima, Hiroshima 739-8526, Japan

×These authors contributed equally to this work

\section{Table of Contents}

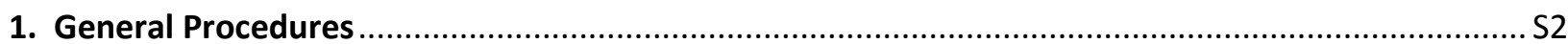

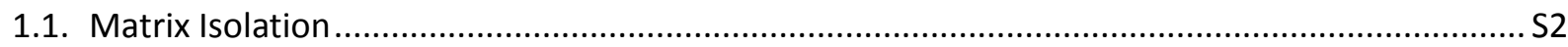

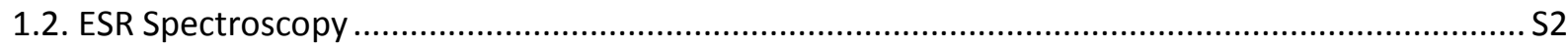

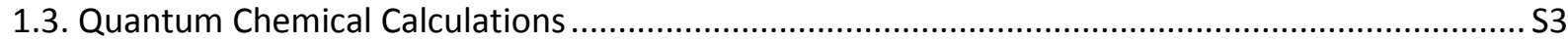

2. Preparation of 2,3-Diazido-1,4-Naphthoquinone (Azide 1) ....................................................... S3

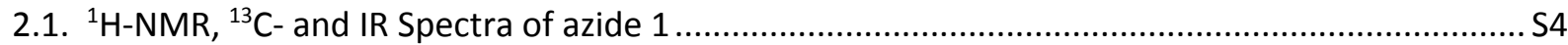

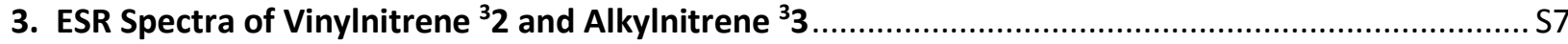

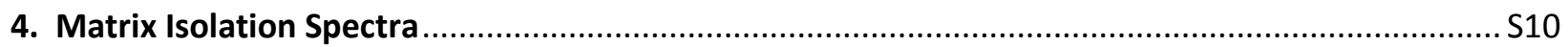

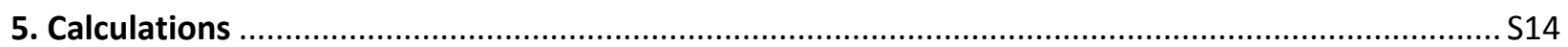

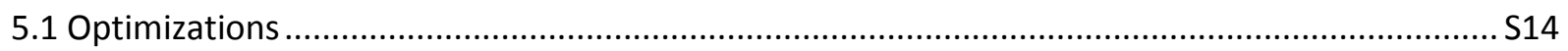

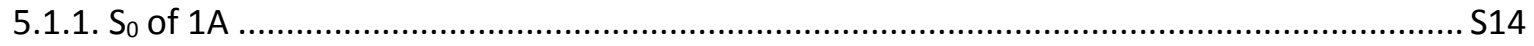

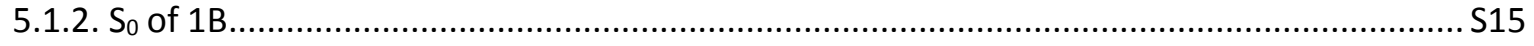

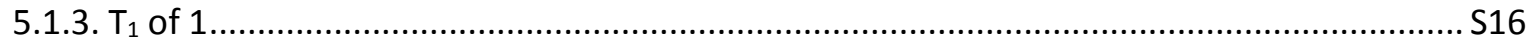

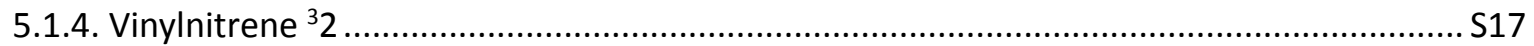

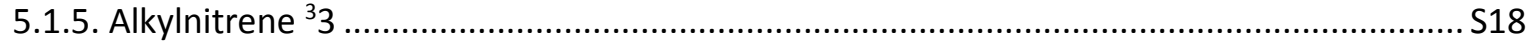

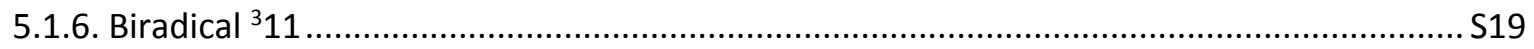

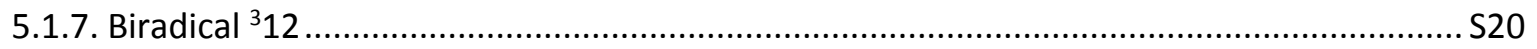

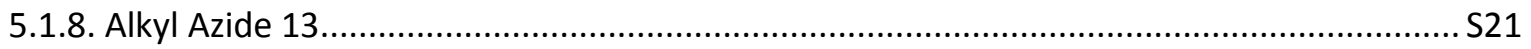

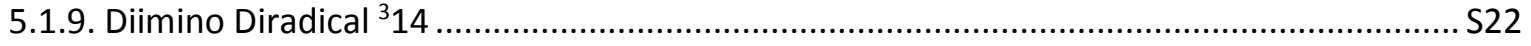


5.1.10. Optimization of $\mathrm{N}_{2}$ S22

5.2. Transition States Optimizations and IRC calculations

5.2.1 Transition state for $T$ of 1 forming vinylnitrene ${ }^{3} 2$ S23

5.2.2. Transition state for vinylnitrene ${ }^{3} 2$ forming biradical ${ }^{3} 11$ S24

5.2.3. Transition state for biradical ${ }^{3} 11$ forming biradical ${ }^{3} 12$.. S25

5.2.4. Transition state for biradical ${ }^{3} 12$ forming alkylnitrene ${ }^{3} 2$ S26

5.2.5. Transition state for vinylnitrene ${ }^{3} 2$ forming triplet diimino diradical ${ }^{3} 14$ S27

5.2.6. Transition state diimino diradical ${ }^{3} 14$ forming biradical ${ }^{3} 12$ S28

5.3. Calculated spin densities of $1, T_{1}$ of 1 , vinylnitrene ${ }^{3} 2$, and alkylnitrene ${ }^{3} 3$. S29

6. References S29

\section{General Procedures}

\subsection{Matrix Isolation}

Argon cooled to $20 \mathrm{~K}$ was deposited for $5 \mathrm{~min}$ on a Csl surface inside the cryostat cold head. Diazides 1 and 2 were sublimed at $40^{\circ} \mathrm{C}$ and subsequently deposited with argon at $20 \mathrm{~K}$ for $40 \mathrm{~min}$ in the vacuum of a diffusion pump. Following the deposition, the cryostat cold head was cooled to $10 \mathrm{~K}$ before irradiating the newly formed matrix. The matrix was irradiated through quartz windows using xenon lamp attached with a filter that only allows $360-440 \mathrm{~nm}$ wavelengths to pass through and IR spectra were recorded after various irradiation times.

\subsection{ESR Spectroscopy}

Diazide 1 was dissolved in anhydrous MTHF and placed in an ESR tube and the resulting solution was degassed by bubbling nitrogen through the solution for 10 minutes, and the tube was capped and sealed with parafilm. Diazide 2 dissolved in EPA, a solvent mixture consisting of two parts ethanol, five parts diethyl ether, and five parts iso-pentane. The resulting solution was placed in an ESR tube and degassed for 10 minutes by bubbling nitrogen through the solution. The tube was capped and wrapped with parafilm. ESR spectra were obtained on a Bruker instrument at Hiroshima University in Japan, which has 
been described in detail elsewhere. Irradiation was performed by using a Xenon lamp attached with a 360-440 $\mathrm{nm}$ band filter.

\subsection{Quantum Chemical Calculations}

Using the Gaussian09 program, geometry optimizations were performed at the B3LYP level of theory

with $6-31+G(d)$ basis set. ${ }^{1-3}$ Absorption spectra were calculated using time-dependent density functional theory (TD-DFT) calculations. ${ }^{4-6}$ All transition states were confirmed to have one imaginary vibrational frequency by the analytical determination of the second derivative of the energy with respect to the internal coordinates. Intrinsic reaction coordinate calculations were used to verify that the transition state correlates with the products and the starting material., ${ }^{7,8}$ The DFT calculations were performed at the Ohio Supercomputer Center.

\section{Preparation of 2,3-Diazido-1,4-Naphthoquinone (Azide 1)}

2,3-Dibromo-1,4-naphthoquinone (1.000 g, $3.175 \mathrm{mmol})$ was dissolved in $40 \mathrm{~mL}$ of acetone and sodium azide $(0.413 \mathrm{~g}, 6.350 \mathrm{mmol})$ was added to the solution in small portions. The reaction mixture was stirred for $24 \mathrm{~h}$ and acetone was removed under reduced pressure at room temperature and the resulting solid was dissolved in diethyl ether. The ether solution was washed several times with water and dried over magnesium sulfate. The ether layer was removed under reduced pressure at room temperature and recrystallized with boiling acetone to yield pure orange crystals $(0.691 \mathrm{~g}, 2.879 \mathrm{mmol}, 91 \%$ yield).

MP: 126 - $129^{\circ} \mathrm{C}$ (lit.: $\left.136^{\circ} \mathrm{C}\right) ;{ }^{9}{ }^{1} \mathrm{H}$ NMR $\left(400 \mathrm{MHz}, \mathrm{CDCl}_{3}\right): \delta 8.11-8.09(\mathrm{~m}, 2 \mathrm{H}), 7.78-7.76(\mathrm{~m}, 2 \mathrm{H}) \mathrm{ppm}$;

${ }^{13}$ C-NMR 179.27, 134.46, 130.83, 129.61, 126.86 ppm. IR (neat): 3024, 2099, 2055, 1657, 1584, 1335, $1300,1172,907,796,738,707 \mathrm{~cm}^{-1}$ 
2.1. ${ }^{1} \mathrm{H}-\mathrm{NMR},{ }^{13} \mathrm{C}$ - and IR Spectra of azide 1

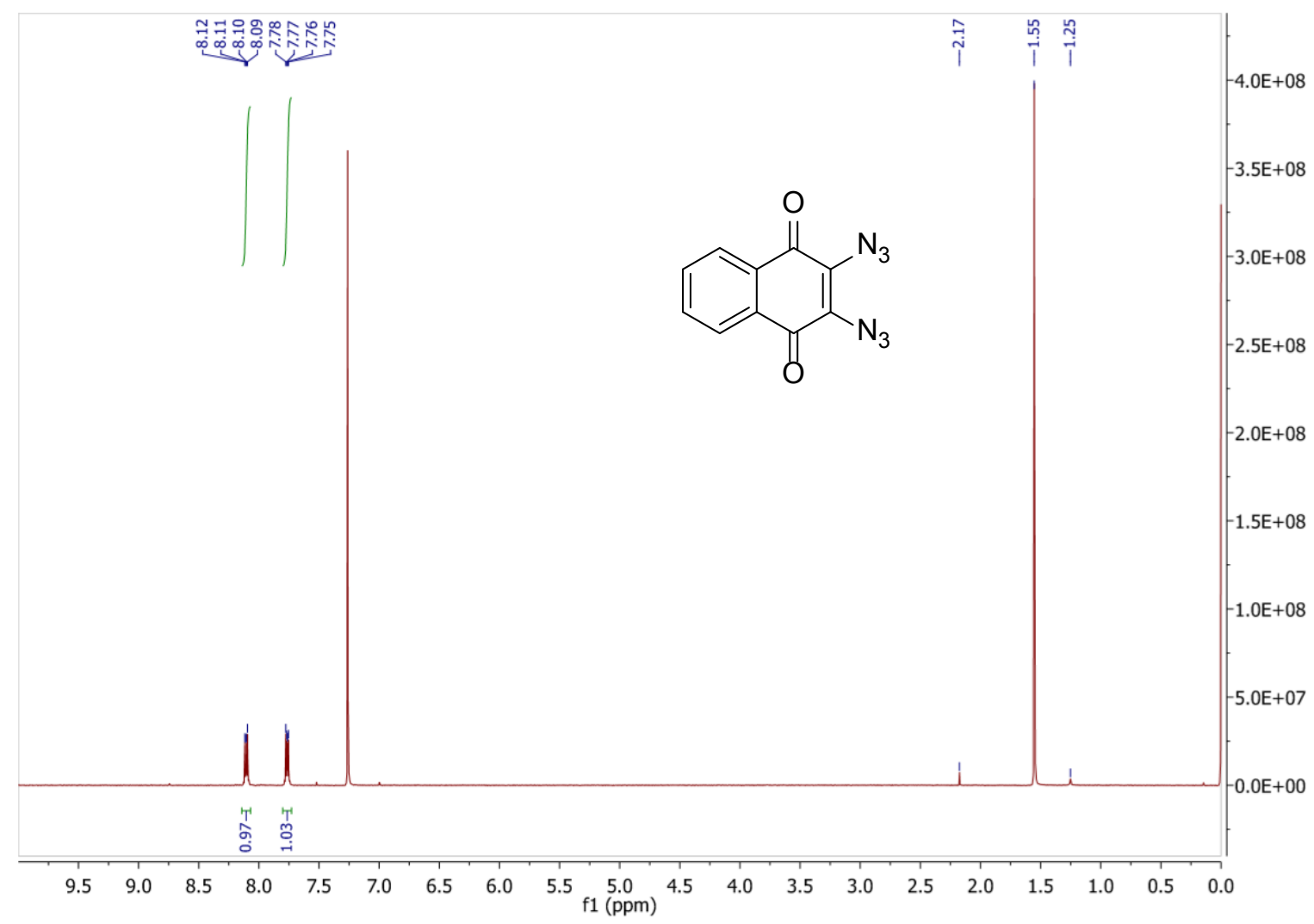

Figure S1: 2,3-Diazido-1,4-Naphthoquinone ${ }^{1} \mathrm{H}-\mathrm{NMR}$ spectrum 


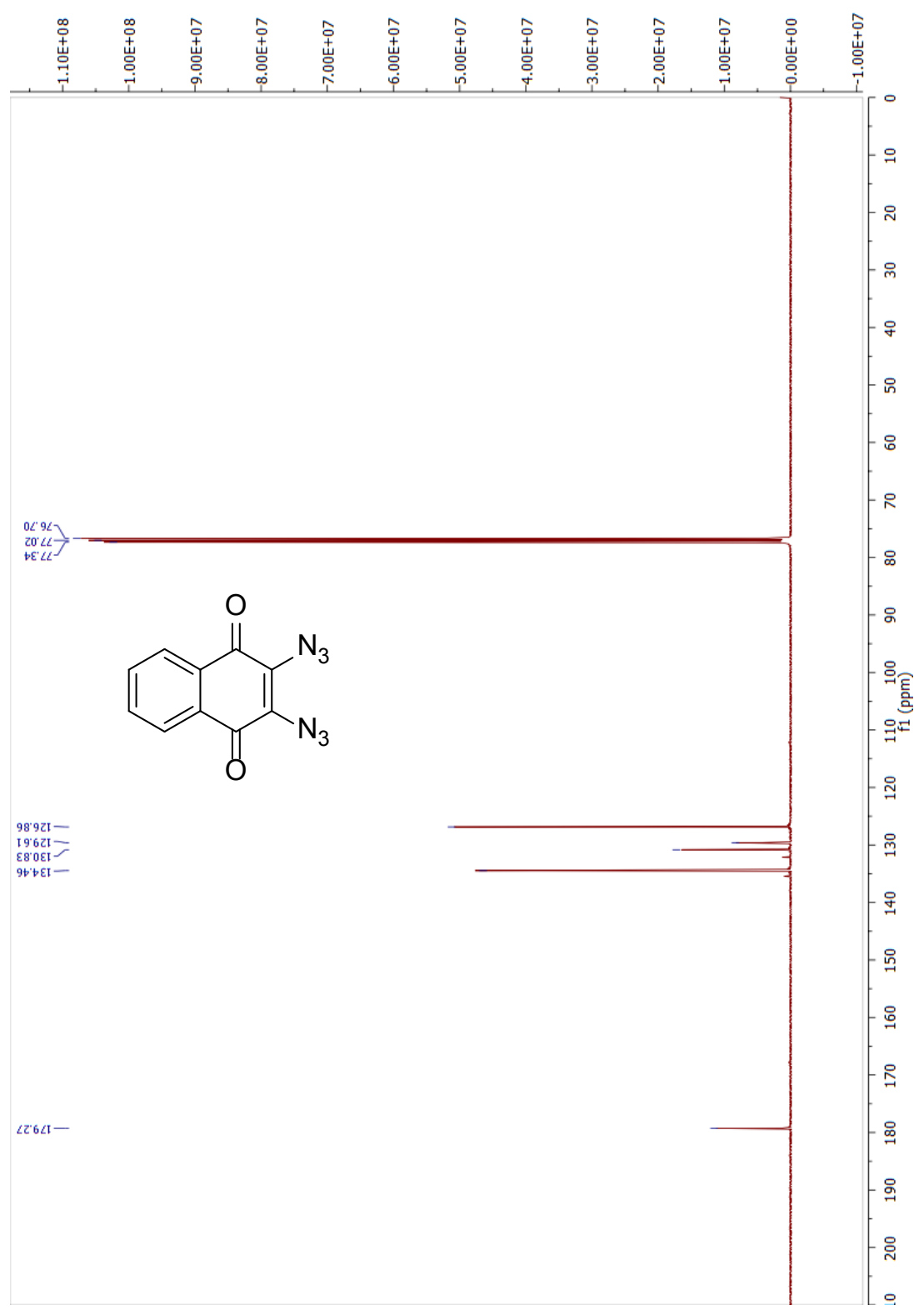

Figure S2: 2,3-Diazido-1,4-Naphthoquinone ${ }^{13} \mathrm{C}-\mathrm{NMR}$ spectrum 


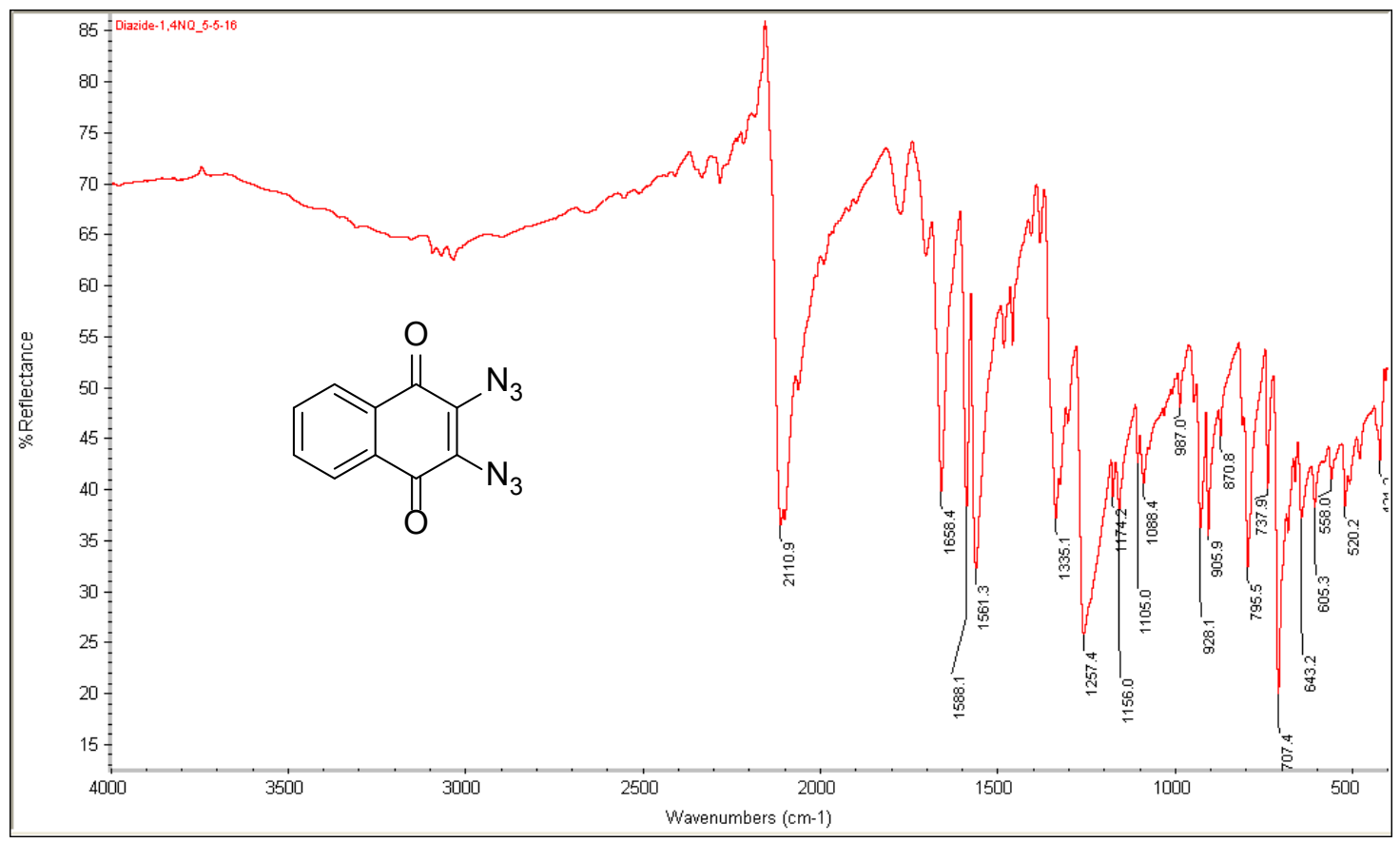

Figure S3: 2,3-Diazido-1,4-Naphthoquinone IR spectra 


\section{ESR Spectra of Vinylnitrene ${ }^{3} \mathbf{2}$ and Alkylnitrene ${ }^{3} 3$}
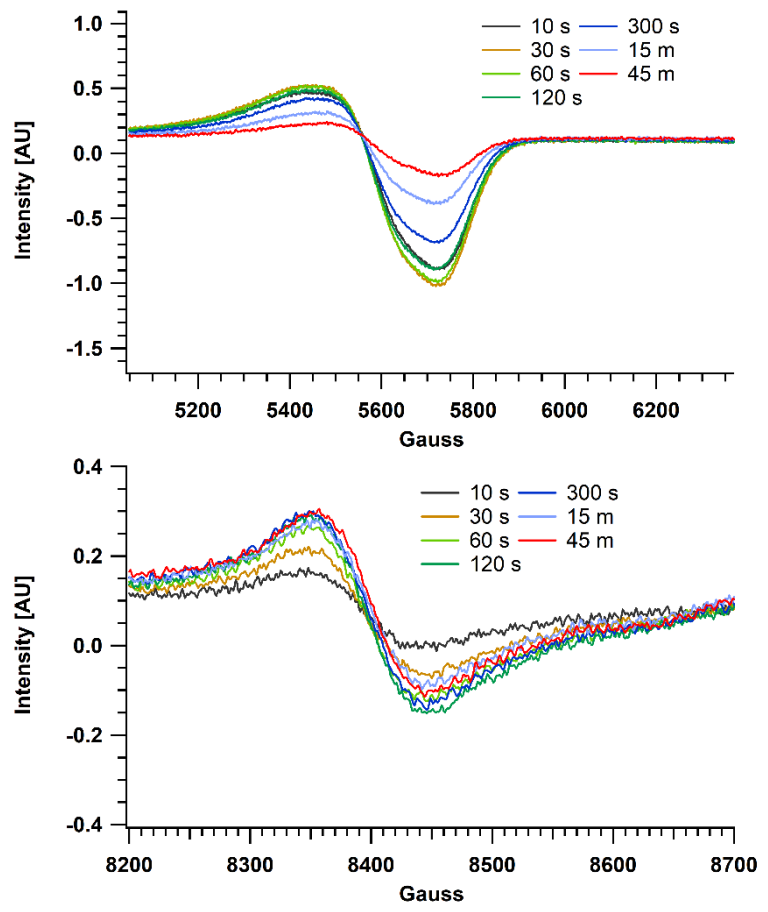

Figure S4: ESR Spectra of vinylnitrene ${ }^{3} \mathbf{2}$ and alkylnitrene ${ }^{3} \mathbf{3}$ as a function of irradiation time. 

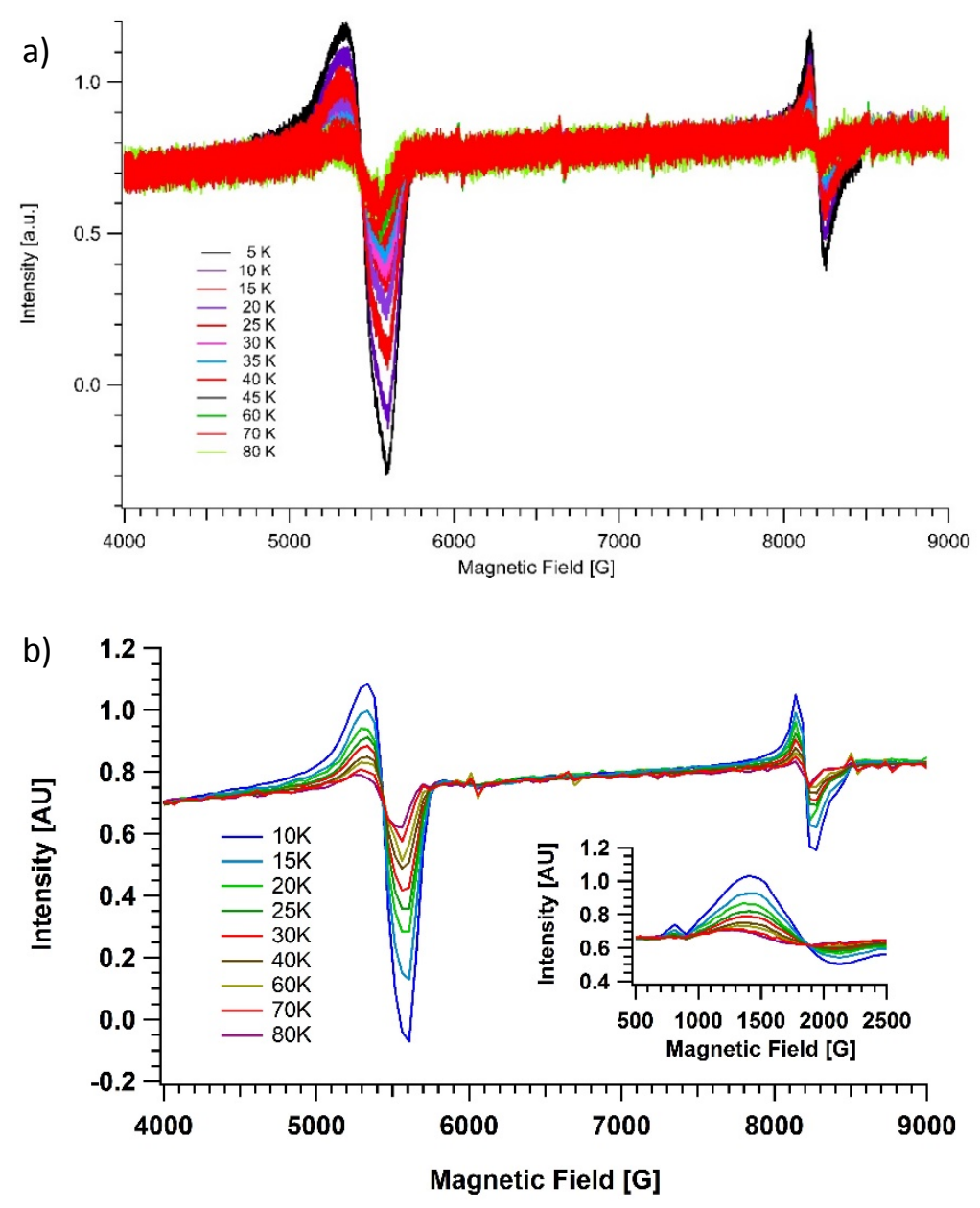

Figure S5: a) ESR Spectra of vinylnitrene ${ }^{3} \mathbf{2}$ and alkylnitrene ${ }^{3} \mathbf{3}$ as a function of temperature, b) with noise reduction. 


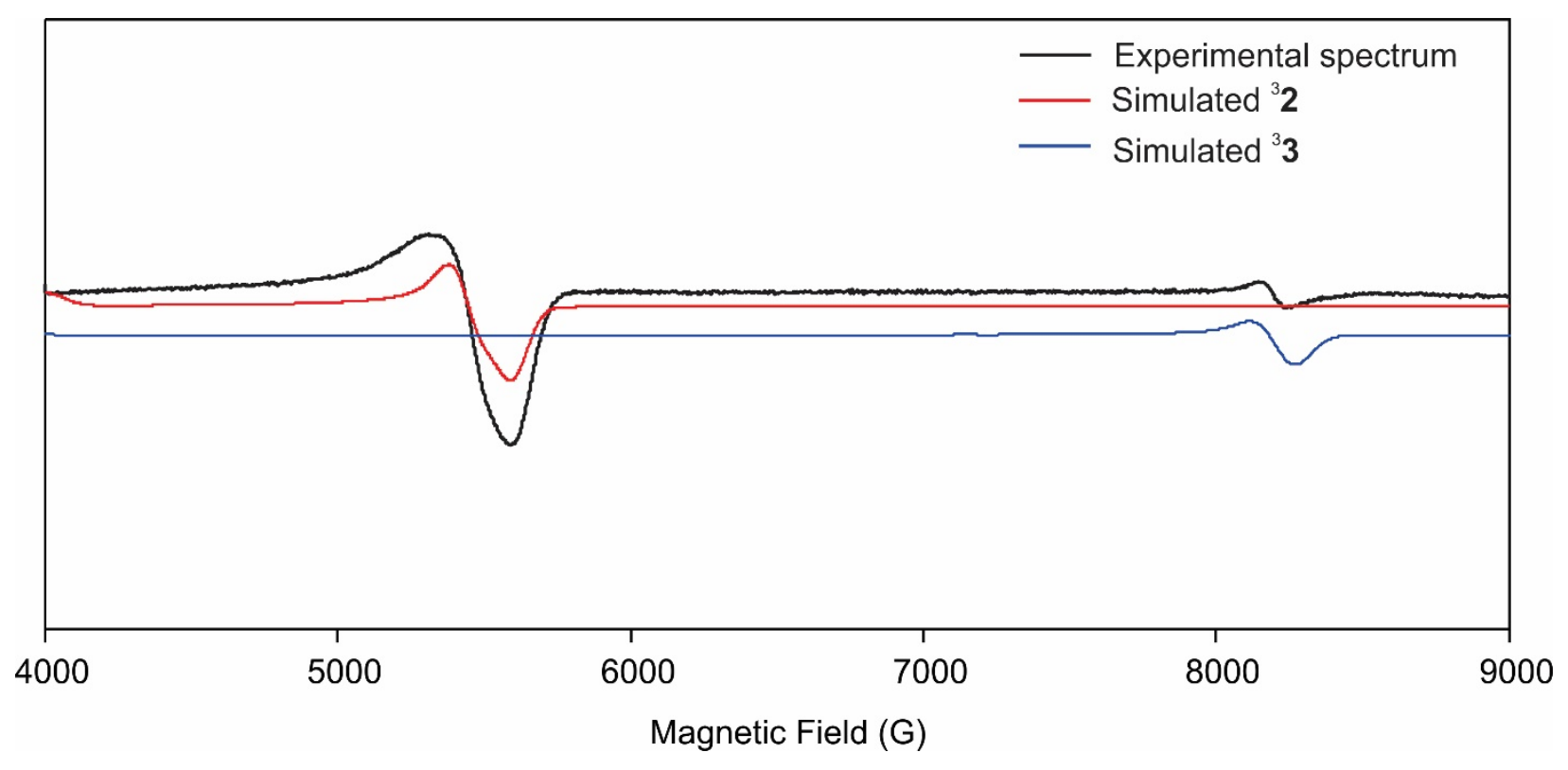

Figure S6: Comparison of the simulated EPR spectra of the vinylnitrene ${ }^{3} \mathbf{2}$ and alkylnitrene ${ }^{3} \mathbf{3}$ with the experimental spectrum. 


\section{Matrix Isolation Spectra}

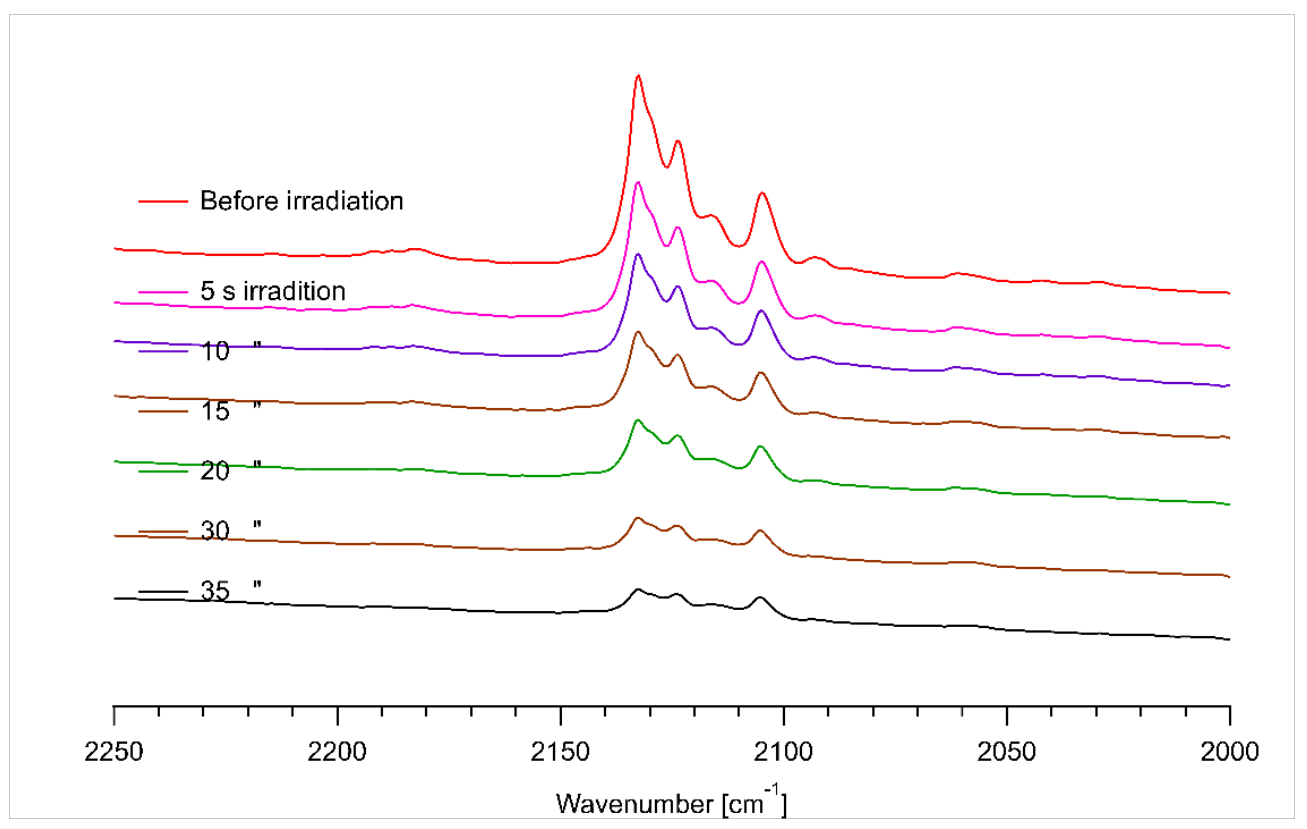

Figure S7. IR spectra between 2250 to $2000 \mathrm{~cm}^{-1}$ of vinyl azide 1 as a function of irradiation time in an argon matrix at $10 \mathrm{~K}$. The matrix was irradiated with xenon lamp through a $360-440 \mathrm{~nm}$ band filter. 


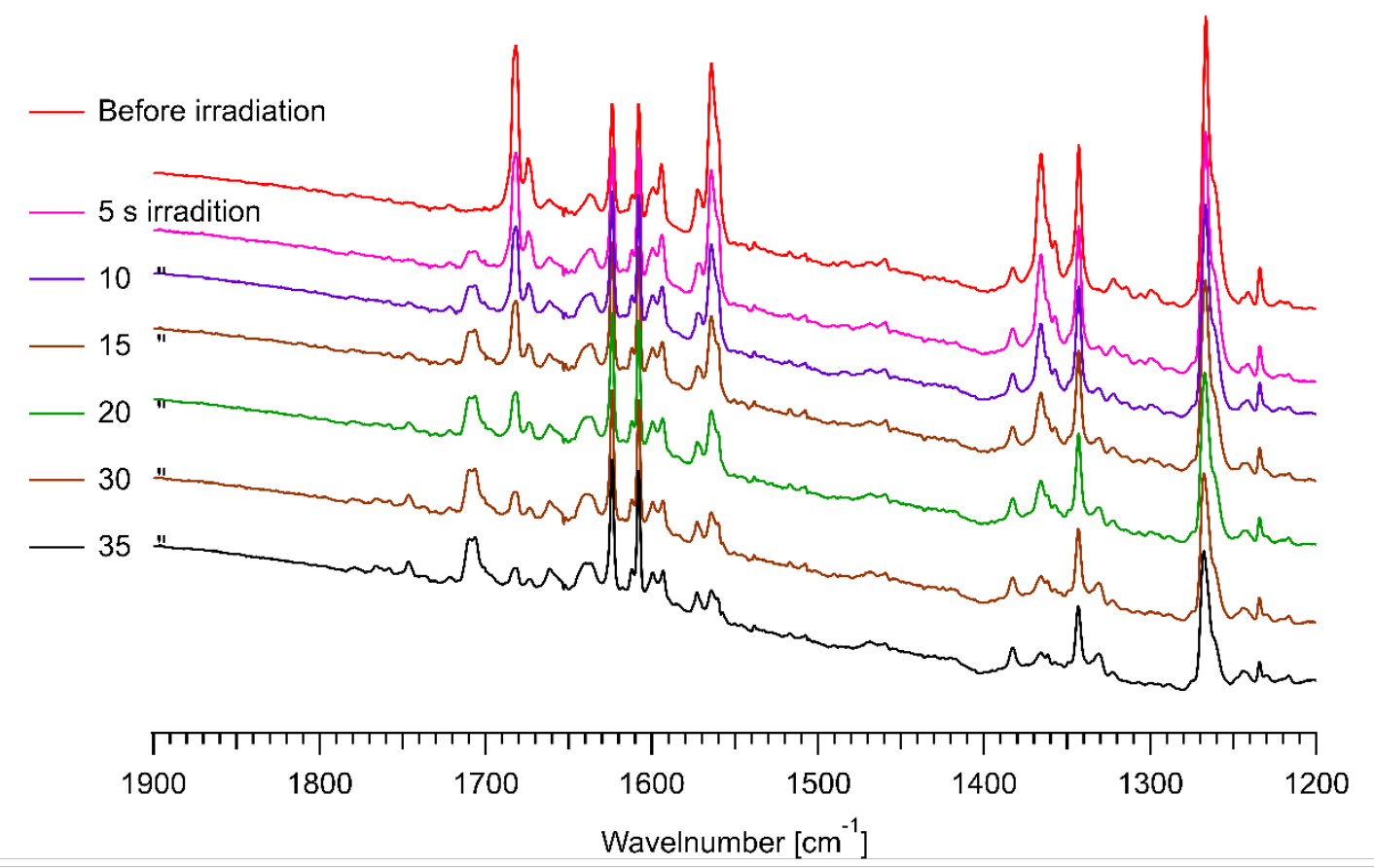

- Before irradiation
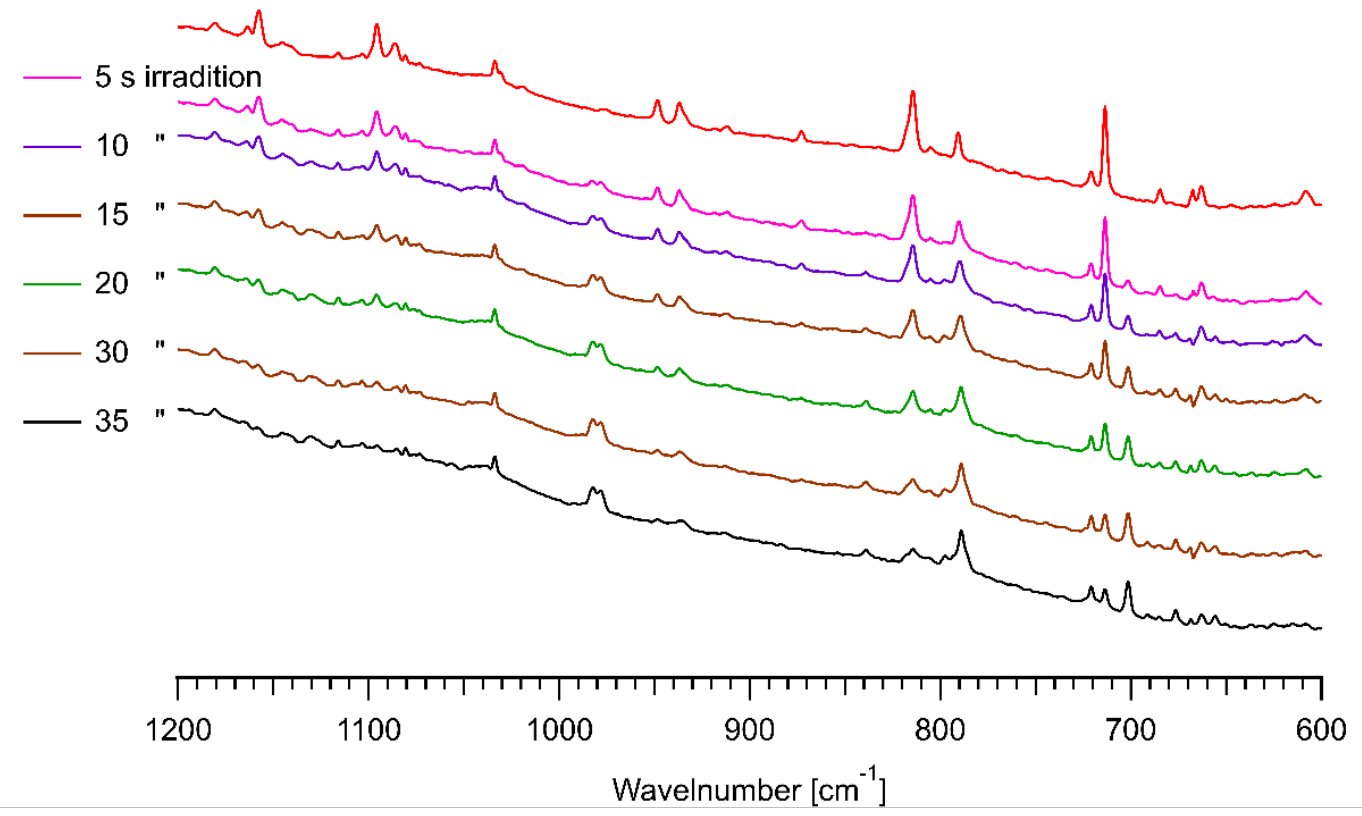

Figure S8. IR spectra of vinyl azide 1 in argon matrix as a function of irradiation time in an argon matrix at $10 \mathrm{~K}$. The matrix was irradiated with xenon lamp through a 360-440 $\mathrm{nm}$ band filter. 


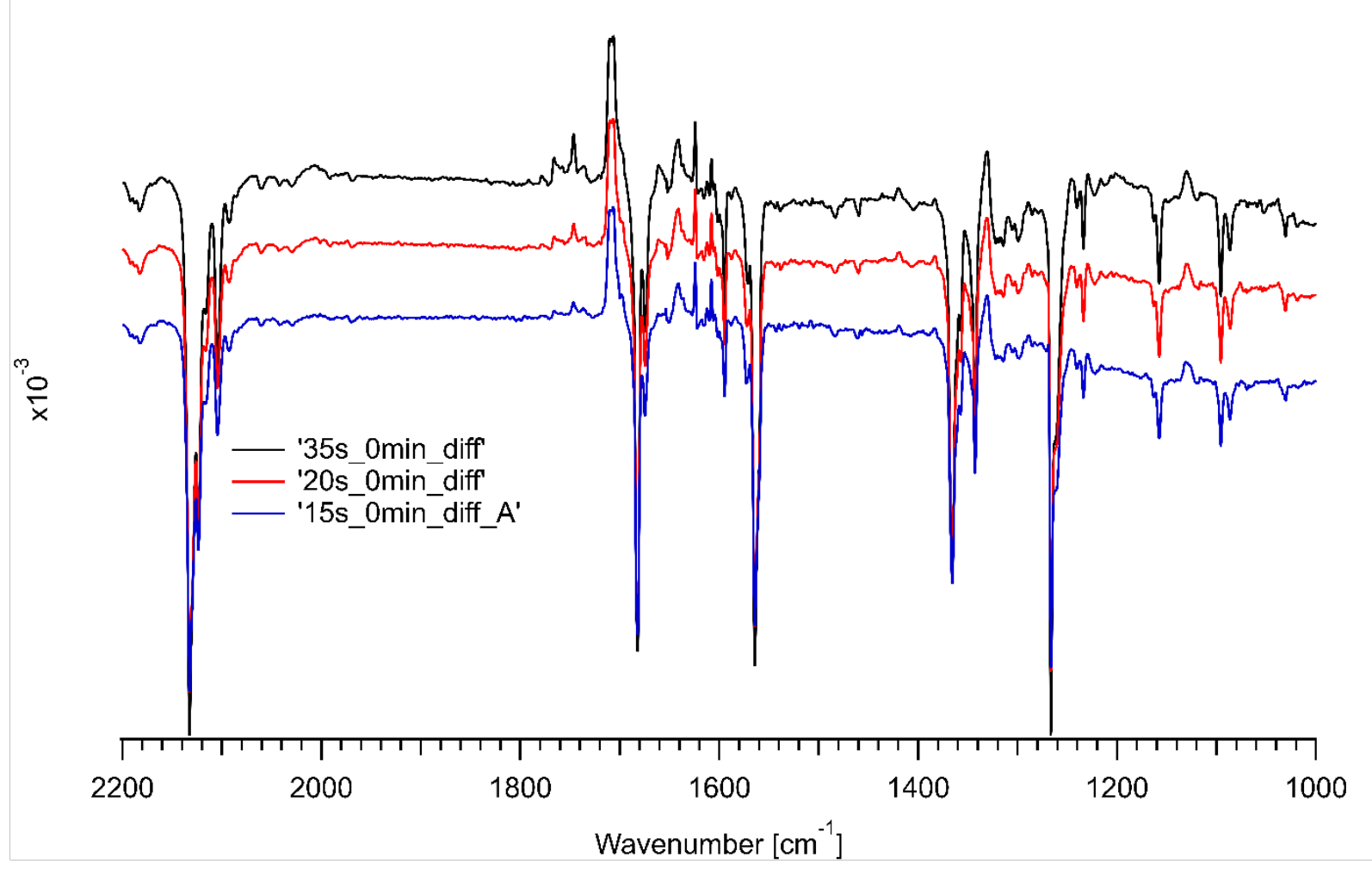

Figure S9. IR difference spectra as function of irradiation time in an argon matrix at $10 \mathrm{~K}$. The matrix was irradiated with xenon lamp through a 360-440 $\mathrm{nm}$ band filter. 


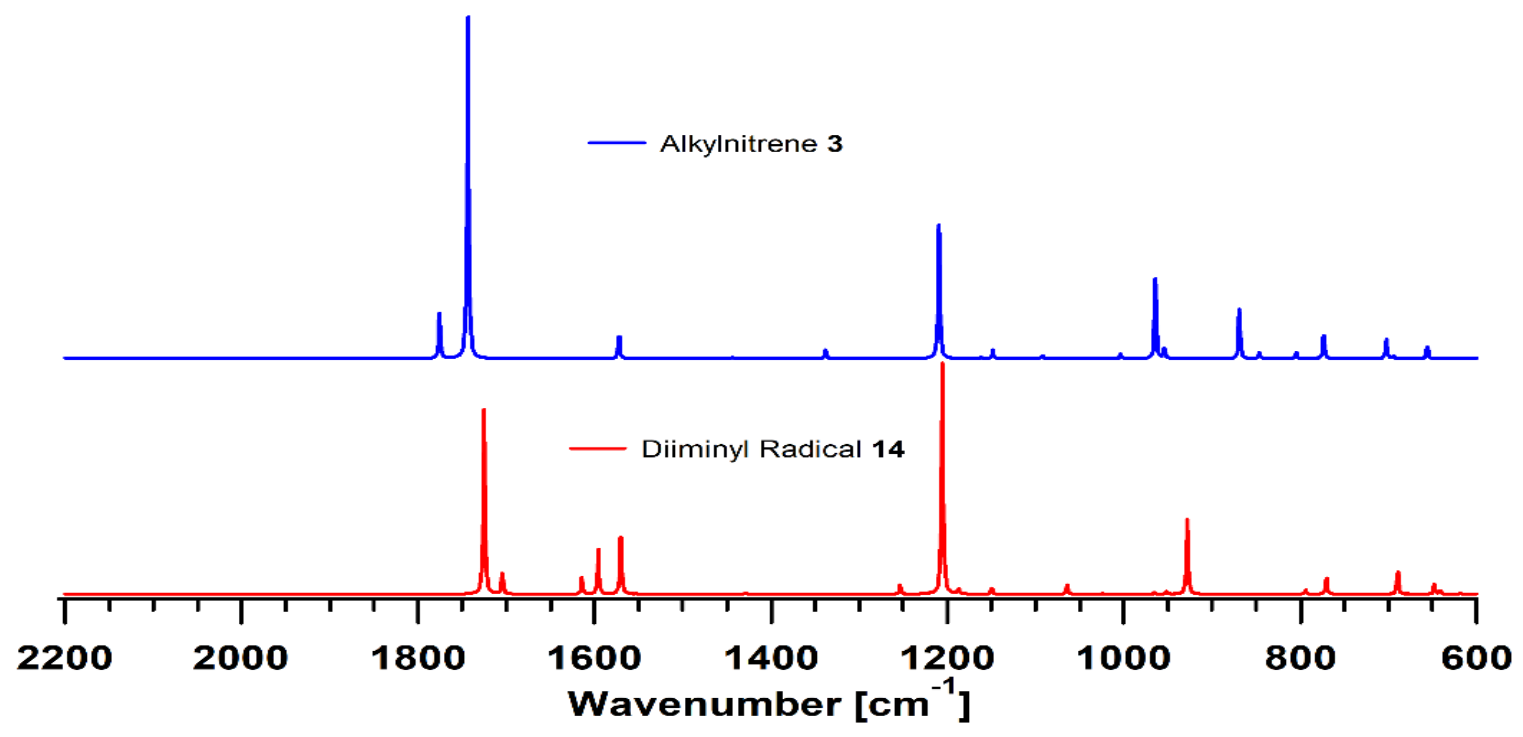

Figure S10. Comparison on the calculated and scaled spectra of alkylnitrene ${ }^{3} \mathbf{3}$ (blue) and diimino diradical ${ }^{3} 14$ (red). 


\section{Calculations}

\subsection{Optimizations}

5.1.1. So of $1 \mathrm{~A}$

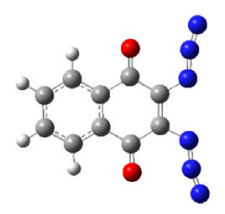

DFT/B3LYP 6-31+G(d), E = -862.326051 a.u.

Standard orientation:

\begin{tabular}{|c|c|c|c|c|c|}
\hline \multirow{3}{*}{$\begin{array}{l}\text { Center } \\
\text { Number } \\
-1 \\
-1\end{array}$} & \multirow{2}{*}{\multicolumn{2}{|c|}{$\begin{array}{l}\text { Atomic } \\
\text { Number }\end{array}$}} & \multirow{2}{*}{$\begin{array}{c}\text { Atomic } \\
r \quad \text { Type }\end{array}$} & \multicolumn{2}{|c|}{ Coordinates (Angstroms } \\
\hline & & & & $\mathrm{Y}$ & Z \\
\hline & & 6 & 2.692445 & 1.401620 & 0.000026 \\
\hline 2 & & 6 & 1.481464 & 0.703112 & -0.000018 \\
\hline 3 & & 6 & 1.481406 & -0.703250 & -0.000015 \\
\hline 4 & & 6 & 2.692333 & -1.401854 & 0.000001 \\
\hline 5 & & 6 & 3.899670 & -0.699664 & 0.000043 \\
\hline 6 & & 6 & 3.899726 & 0.699335 & 0.000054 \\
\hline 7 & & 0 & 2.672017 & 2.486776 & 0.000032 \\
\hline 8 & & 1 & 2.671818 & -2.487008 & -0.000021 \\
\hline 9 & & 1 & 4.840141 & -1.243754 & 0.000063 \\
\hline 10 & & 1 & 4.840241 & 1.243349 & 0.000086 \\
\hline 11 & & 6 & 0.199051 & -1.457475 & -0.000061 \\
\hline 12 & & 8 & 0.167639 & -2.685236 & -0.000087 \\
\hline 13 & & 6 & 0.199167 & 1.457429 & -0.000028 \\
\hline 14 & & 8 & 0.167823 & 2.685197 & 0.000012 \\
\hline 15 & & 7 & -2.296787 & -1.336283 & -0.000006 \\
\hline 16 & & 6 & -1.072644 & -0.688982 & -0.000063 \\
\hline 17 & & 7 & -2.391131 & -2.582028 & 0.000065 \\
\hline 18 & & 7 & -2.748876 & -3.660560 & 0.000137 \\
\hline 19 & & 6 & -1.072591 & 0.689050 & -0.000094 \\
\hline 20 & & 7 & -2.296664 & 1.336483 & -0.000126 \\
\hline 21 & & 7 & -2.390792 & 2.582251 & 0.000015 \\
\hline 22 & & 7 & -2.748333 & 3.660852 & 0.000111 \\
\hline
\end{tabular}




\subsubsection{So of $1 B$}

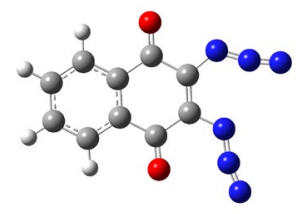

DFT/B3LYP 6-31+G(d), E = -862.321819 a.u.

Standard orientation:

\begin{tabular}{|c|c|c|c|c|c|}
\hline \multirow{2}{*}{\multicolumn{2}{|c|}{$\begin{array}{l}\text { Center } \\
\text { Number }\end{array}$}} & \multirow{2}{*}{\multicolumn{2}{|c|}{$\begin{array}{c}\text { Atomic Atomic } \\
\text { Number Type }\end{array}$}} & \multicolumn{2}{|c|}{ Coordinates (Angst } \\
\hline & & & & $x$ & $Y$ \\
\hline 1 & 6 & 0 & -2.655529 & -1.546672 & 0.000072 \\
\hline 2 & 6 & 0 & -1.550429 & -0.688510 & 0.000024 \\
\hline 3 & 6 & 0 & -1.739459 & 0.705019 & -0.000023 \\
\hline 4 & 6 & 0 & -3.035170 & 1.230347 & -0.000015 \\
\hline 5 & 6 & 0 & -4.135558 & 0.370877 & 0.000032 \\
\hline 6 & 6 & 0 & -3.946329 & -1.015848 & 0.000077 \\
\hline 7 & 1 & 0 & -2.487084 & -2.618869 & 0.000107 \\
\hline 8 & 1 & 0 & -3.161504 & 2.308294 & -0.000048 \\
\hline 9 & 1 & 0 & -5.141274 & 0.782168 & 0.000034 \\
\hline 10 & 1 & 0 & -4.804351 & -1.682436 & 0.000115 \\
\hline 11 & 6 & 0 & -0.573322 & 1.634877 & -0.000069 \\
\hline 12 & 8 & 0 & -0.721472 & 2.848842 & -0.000084 \\
\hline 13 & 6 & 0 & -0.180775 & -1.261319 & 0.000000 \\
\hline 14 & 8 & 0 & 0.024837 & -2.472495 & 0.000049 \\
\hline 15 & 7 & 0 & 1.781779 & 2.014081 & -0.000057 \\
\hline 16 & 6 & 0 & 0.797889 & 1.036303 & -0.000102 \\
\hline 17 & 7 & 0 & 2.999073 & 1.754199 & 0.000106 \\
\hline 18 & 7 & 0 & 4.136352 & 1.758885 & 0.000237 \\
\hline 19 & 6 & 0 & 0.976082 & -0.327417 & -0.000055 \\
\hline 20 & 7 & 0 & 2.276426 & -0.813488 & -0.000109 \\
\hline 21 & 7 & 0 & 2.542449 & -2.033488 & -0.000068 \\
\hline 22 & 7 & 0 & 3.038620 & -3.055314 & -0.000048 \\
\hline
\end{tabular}




\subsection{3. $T_{1}$ of 1}

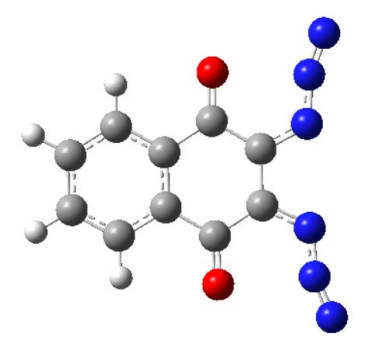

DFT/B3LYP 6-31+G(d), E = -862.27900054 a.u.

Standard orientation:

\begin{tabular}{|c|c|c|c|c|c|}
\hline \multirow{2}{*}{$\begin{array}{l}\text { Center } \\
\text { Number }\end{array}$} & \multicolumn{2}{|c|}{ Atomic } & \multirow{2}{*}{$\begin{array}{r}\text { Atomic } \\
\text { Type }\end{array}$} & \multicolumn{2}{|c|}{ Coordinates (Angstroms } \\
\hline & & Jumber & & Y & Z \\
\hline 1 & 6 & 0 & 2.674591 & 1.402066 & 0.000018 \\
\hline 2 & 6 & 0 & 1.447978 & 0.710205 & 0.000009 \\
\hline 3 & 6 & 0 & 1.447978 & -0.710205 & 0.000003 \\
\hline 4 & 6 & 0 & 2.674591 & -1.402066 & 0.000006 \\
\hline 5 & 6 & 0 & 3.873714 & -0.704145 & 0.000015 \\
\hline 6 & 6 & 0 & 3.873713 & 0.704145 & 0.000021 \\
\hline 7 & 1 & 0 & 2.653326 & 2.487189 & 0.000023 \\
\hline 8 & 1 & 0 & 2.653326 & -2.487189 & 0.000001 \\
\hline 9 & 1 & 0 & 4.815661 & -1.246107 & 0.000018 \\
\hline 10 & 1 & 0 & 4.815661 & 1.246107 & 0.000028 \\
\hline 11 & 6 & 0 & 0.197609 & -1.482367 & -0.000008 \\
\hline 12 & 8 & 0 & 0.148578 & -2.732883 & -0.000013 \\
\hline 13 & 6 & 0 & 0.197609 & 1.482368 & 0.000007 \\
\hline 14 & 8 & 0 & 0.148578 & 2.732883 & 0.000011 \\
\hline 15 & 7 & 0 & -2.253924 & -1.340777 & -0.000004 \\
\hline 16 & 6 & 0 & -1.044671 & -0.731247 & -0.000008 \\
\hline 17 & 7 & 0 & -2.348287 & -2.602052 & -0.000005 \\
\hline 18 & 7 & 0 & -2.762494 & -3.660686 & -0.000005 \\
\hline 19 & 6 & 0 & -1.044671 & 0.731247 & -0.000006 \\
\hline 20 & 7 & 0 & -2.253924 & 1.340777 & -0.000021 \\
\hline 21 & 7 & 0 & -2.348288 & 2.602051 & -0.000013 \\
\hline 22 & 7 & 0 & -2.762495 & 3.660685 & -0.000011 \\
\hline
\end{tabular}




\subsubsection{Vinylnitrene ${ }^{3} 2$}

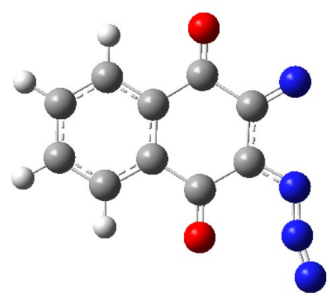

DFT/B3LYP 6-31+G(d), E = -752.79950389

Standard orientation:

\begin{tabular}{|c|c|c|c|c|c|}
\hline \multirow{2}{*}{$\begin{array}{l}\text { Center } \\
\text { Number }\end{array}$} & Atomic & \multicolumn{2}{|c|}{ Atomic } & \multicolumn{2}{|c|}{ Coordinates (Angstrom } \\
\hline & Numb & & Type & Y & Z \\
\hline 1 & 6 & 0 & 1.578020 & -1.992008 & 0.000130 \\
\hline 2 & 6 & 0 & 0.848684 & -0.795962 & 0.000054 \\
\hline 3 & 6 & 0 & 1.530821 & 0.438996 & 0.000001 \\
\hline 4 & 6 & 0 & 2.931856 & 0.459506 & 0.000020 \\
\hline 5 & 6 & 0 & 3.649508 & -0.735364 & 0.000099 \\
\hline 6 & 6 & 0 & 2.971725 & -1.961325 & 0.000154 \\
\hline 7 & 1 & 0 & 1.035976 & -2.932131 & 0.000166 \\
\hline 8 & 1 & 0 & 3.437257 & 1.419939 & -0.000025 \\
\hline 9 & 1 & 0 & 4.735763 & -0.713553 & 0.000118 \\
\hline 10 & 1 & 0 & 3.531871 & -2.892364 & 0.000215 \\
\hline 11 & 6 & 0 & 0.799651 & 1.736590 & -0.000085 \\
\hline 12 & 8 & 0 & 1.374136 & 2.809162 & -0.000210 \\
\hline 13 & 6 & 0 & -0.636225 & -0.867105 & 0.000018 \\
\hline 14 & 8 & 0 & -1.244920 & -1.942012 & 0.000022 \\
\hline 15 & 7 & 0 & -1.393192 & 2.783790 & 0.000084 \\
\hline 16 & 6 & 0 & -0.734458 & 1.680960 & 0.000005 \\
\hline 17 & 6 & 0 & -1.389923 & 0.391011 & -0.000024 \\
\hline 18 & 7 & 0 & -2.755916 & 0.412726 & -0.000072 \\
\hline 19 & 7 & 0 & -3.438842 & -0.641182 & -0.000083 \\
\hline 20 & 7 & 0 & -4.279557 & -1.405460 & -0.000102 \\
\hline
\end{tabular}




\subsubsection{Alkylnitrene ${ }^{3} 3$}

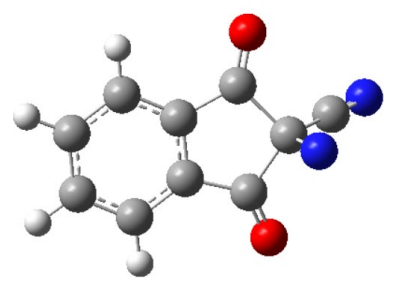

DFT/B3LYP 6-31+G(d), E = -643.30455422 a.u.

Standard orientation:

\begin{tabular}{|c|c|c|c|c|c|}
\hline \multirow{2}{*}{$\begin{array}{l}\text { Center } \\
\text { Number }\end{array}$} & \multicolumn{2}{|c|}{ Atomic } & Atomic & \multicolumn{2}{|c|}{ Coordinates (Angstron } \\
\hline & & Number & Type & Y & Z \\
\hline 1 & 6 & 0 & 2.691528 & 0.000009 & -0.513025 \\
\hline 2 & 6 & 0 & 0.549365 & -1.243484 & 0.096127 \\
\hline 3 & 6 & 0 & -0.823492 & -0.700995 & 0.009406 \\
\hline 4 & 6 & 0 & -0.823492 & 0.700993 & 0.009406 \\
\hline 5 & 6 & 0 & 0.549359 & 1.243483 & 0.096123 \\
\hline 6 & 6 & 0 & 1.519202 & 0.000003 & 0.360973 \\
\hline 7 & 1 & 0 & -2.001443 & -2.509066 & -0.100458 \\
\hline 8 & 6 & 0 & -2.014416 & -1.423409 & -0.095475 \\
\hline 9 & 6 & 0 & -2.014417 & 1.423407 & -0.095470 \\
\hline 10 & 6 & 0 & -3.206371 & 0.703779 & -0.191438 \\
\hline 11 & 6 & 0 & -3.206371 & -0.703782 & -0.191441 \\
\hline 12 & 1 & 0 & -2.001445 & 2.509065 & -0.100448 \\
\hline 13 & 1 & 0 & -4.150426 & 1.235721 & -0.270273 \\
\hline 14 & 1 & 0 & -4.150425 & -1.235724 & -0.270280 \\
\hline 15 & 8 & 0 & 0.911670 & 2.388647 & 0.010161 \\
\hline 16 & 8 & 0 & 0.911679 & -2.388650 & 0.010168 \\
\hline 17 & 7 & 0 & 3.616248 & -0.000003 & -1.214958 \\
\hline 18 & 7 & 0 & 1.868264 & 0.000005 & 1.738918 \\
\hline
\end{tabular}


5.1.6. Biradical ${ }^{3} 11$

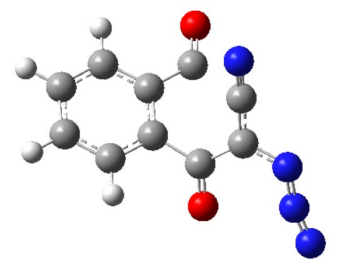

DFT/B3LYP 6-31+G(d), E = -752.77091581 a.u.

Standard orientation:

\begin{tabular}{|c|c|c|c|c|c|}
\hline \multirow{2}{*}{$\begin{array}{l}\text { Center } \\
\text { Number }\end{array}$} & \multicolumn{2}{|l|}{ Atomic } & Atomic & \multicolumn{2}{|c|}{ Coordinates (Angstroms) } \\
\hline & Numb & & Type & Y & Z \\
\hline 1 & 6 & 0 & -2.978148 & 0.299194 & -0.330762 \\
\hline 2 & 6 & 0 & -1.580467 & 0.397964 & -0.441125 \\
\hline 3 & 6 & 0 & -0.764524 & -0.675576 & -0.029120 \\
\hline 4 & 6 & 0 & -1.362976 & -1.841608 & 0.465149 \\
\hline 5 & 6 & 0 & -2.750813 & -1.925055 & 0.584883 \\
\hline 6 & 6 & 0 & -3.560225 & -0.853636 & 0.190458 \\
\hline 7 & 1 & 0 & -3.588931 & 1.131874 & -0.666753 \\
\hline 8 & 1 & 0 & -0.734964 & -2.677558 & 0.758157 \\
\hline 9 & 1 & 0 & -3.201854 & -2.830380 & 0.981746 \\
\hline 10 & 1 & 0 & -4.640776 & -0.923042 & 0.281195 \\
\hline 11 & 6 & 0 & 0.732316 & -0.665714 & -0.144092 \\
\hline 12 & 8 & 0 & 1.329162 & -1.565397 & -0.739710 \\
\hline 13 & 6 & 0 & -0.981032 & 1.596546 & -1.080581 \\
\hline 14 & 8 & 0 & -1.534433 & 2.575383 & -1.477682 \\
\hline 15 & 7 & 0 & 2.885848 & 0.442095 & 0.427448 \\
\hline 16 & 6 & 0 & 1.525661 & 0.372222 & 0.530931 \\
\hline 17 & 7 & 0 & 3.529206 & -0.351096 & -0.307198 \\
\hline 18 & 7 & 0 & 4.339868 & -0.908510 & -0.876340 \\
\hline 19 & 6 & 0 & 0.948154 & 1.332482 & 1.395718 \\
\hline 20 & 7 & 0 & 0.450938 & 2.102983 & 2.118380 \\
\hline
\end{tabular}




\subsubsection{Biradical ${ }^{3} 12$}

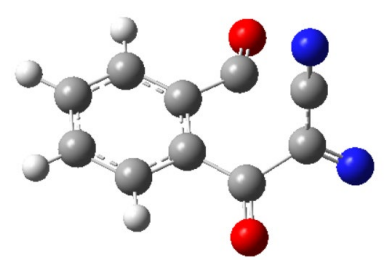

DFT/B3LYP 6-31+G(d), E = -643.28170797 a.u.

Standard orientation:

\begin{tabular}{|c|c|c|c|c|}
\hline \multirow{2}{*}{$\begin{array}{l}\text { Center } \\
\text { Number }\end{array}$} & Atomic & \multirow{2}{*}{$\begin{array}{l}\text { Atomic } \\
r \quad \text { Type }\end{array}$} & \multicolumn{2}{|c|}{ Coordinates (Angstroms) } \\
\hline & Numb & & $Y$ & Z \\
\hline 1 & 6 & -2.127006 & 1.199273 & -0.008159 \\
\hline 2 & 6 & -0.832524 & 0.712213 & -0.252335 \\
\hline 3 & 6 & -0.554657 & -0.665714 & -0.149196 \\
\hline 4 & 6 & -1.593303 & -1.551318 & 0.168746 \\
\hline 5 & 6 & -2.875280 & -1.063102 & 0.419764 \\
\hline 6 & 6 & -3.142703 & 0.309790 & 0.335928 \\
\hline 7 & 1 & -2.319390 & 2.264034 & -0.098368 \\
\hline 8 & 1 & -1.379206 & -2.613641 & 0.226474 \\
\hline 9 & 1 & -3.671048 & -1.754440 & 0.682435 \\
\hline 10 & 1 & -4.145289 & 0.681722 & 0.528146 \\
\hline 11 & 6 & 0.804345 & -1.239745 & -0.392021 \\
\hline 12 & 8 & 0.970990 & -2.387633 & -0.742226 \\
\hline 13 & 6 & 0.256501 & 1.628963 & -0.619603 \\
\hline 14 & 8 & 0.253144 & 2.761871 & -0.973255 \\
\hline 15 & 7 & 3.029818 & -0.345407 & -0.935737 \\
\hline 16 & 6 & 2.055512 & -0.352515 & -0.124147 \\
\hline 17 & 6 & 2.155768 & 0.287551 & 1.170071 \\
\hline 18 & 7 & 2.233315 & 0.750557 & 2.234433 \\
\hline
\end{tabular}




\subsubsection{Alkyl Azide 13}

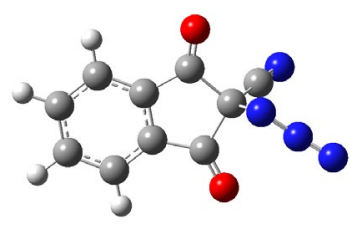

DFT/B3LYP 6-31+G(d), E = -752.847591 a.u.

Standard orientation:

\begin{tabular}{|c|c|c|c|c|c|}
\hline \multirow{2}{*}{$\begin{array}{l}\text { Center } \\
\text { Number }\end{array}$} & \multicolumn{2}{|c|}{ Atomic } & \multirow{2}{*}{$\begin{array}{l}\text { Atomic } \\
\text { Type }\end{array}$} & \multicolumn{2}{|c|}{ Coordinates (Angstrom } \\
\hline & & aber & & $\mathrm{Y}$ & Z \\
\hline 1 & 6 & 0 & -1.706211 & 0.654918 & 1.344407 \\
\hline 2 & 6 & 0 & -0.260935 & -1.037784 & 0.214719 \\
\hline 3 & 6 & 0 & 1.178564 & -0.726568 & 0.094930 \\
\hline 4 & 6 & 0 & 1.375014 & 0.639007 & -0.162109 \\
\hline 5 & 6 & 0 & 0.084615 & 1.359736 & -0.241985 \\
\hline 6 & 6 & 0 & -1.051436 & 0.316448 & 0.075846 \\
\hline 7 & 1 & 0 & 2.095200 & -2.660460 & 0.396148 \\
\hline 8 & 6 & 0 & 2.259722 & -1.605468 & 0.198956 \\
\hline 9 & 6 & 0 & 2.658798 & 1.165324 & -0.323138 \\
\hline 10 & 6 & 0 & 3.740072 & 0.289885 & -0.215445 \\
\hline 11 & 6 & 0 & 3.542931 & -1.080298 & 0.043126 \\
\hline 12 & 1 & 0 & 2.798966 & 2.222890 & -0.525229 \\
\hline 13 & 1 & 0 & 4.751807 & 0.667914 & -0.333508 \\
\hline 14 & 1 & 0 & 4.405753 & -1.735983 & 0.120764 \\
\hline 15 & 8 & 0 & -0.103489 & 2.522850 & -0.503889 \\
\hline 16 & 8 & 0 & -0.789294 & -2.118112 & 0.350752 \\
\hline 17 & 7 & 0 & -2.224240 & 0.909200 & 2.352607 \\
\hline 18 & 7 & 0 & -1.962666 & 0.290575 & -1.076455 \\
\hline 19 & 7 & 0 & -2.977514 & -0.423972 & -0.947928 \\
\hline 20 & 7 & 0 & -3.955046 & -1.002010 & -0.986640 \\
\hline
\end{tabular}




\subsubsection{Diimino Diradical ${ }^{3} 14$}

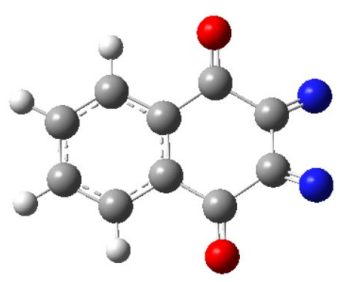

DFT/B3LYP 6-31+G(d), E = -643.29949443 a.u.

Standard orientation:

\begin{tabular}{|c|c|c|c|c|c|}
\hline \multirow{2}{*}{$\begin{array}{l}\text { Center } \\
\text { Number }\end{array}$} & Atomic & \multicolumn{2}{|c|}{ Atomic } & \multicolumn{2}{|c|}{ Coordinates (Angstroms) } \\
\hline & Numb & & Type & $x$ & Z \\
\hline 1 & 6 & 0 & 1.894456 & 1.397396 & -0.079418 \\
\hline 2 & 6 & 0 & 0.673956 & 0.706615 & -0.039026 \\
\hline 3 & 6 & 0 & 0.673961 & -0.706606 & 0.039027 \\
\hline 4 & 6 & 0 & 1.894466 & -1.397378 & 0.079426 \\
\hline 5 & 6 & 0 & 3.098819 & -0.699591 & 0.040699 \\
\hline 6 & 6 & 0 & 3.098814 & 0.699618 & -0.040680 \\
\hline 7 & 1 & 0 & 1.874653 & 2.480952 & -0.135999 \\
\hline 8 & 1 & 0 & 1.874668 & -2.480934 & 0.136003 \\
\hline 9 & 1 & 0 & 4.039037 & -1.243065 & 0.071328 \\
\hline 10 & 1 & 0 & 4.039029 & 1.243099 & -0.071300 \\
\hline 11 & 6 & 0 & -0.579210 & -1.513679 & 0.055200 \\
\hline 12 & 8 & 0 & -0.594041 & -2.717758 & 0.209960 \\
\hline 13 & 6 & 0 & -0.579222 & 1.513681 & -0.055204 \\
\hline 14 & 8 & 0 & -0.594075 & 2.717747 & -0.210028 \\
\hline 15 & 7 & 0 & -2.910757 & -1.288836 & -0.640192 \\
\hline 16 & 6 & 0 & -1.885621 & -0.733212 & -0.163543 \\
\hline 17 & 6 & 0 & -1.885660 & 0.733187 & 0.163605 \\
\hline 18 & 7 & 0 & -2.910816 & 1.288813 & 0.640191 \\
\hline
\end{tabular}

5.1.10. Optimization of $\mathrm{N}_{2}$

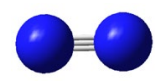

DFT/B3LYP 6-31+G(d), E = -109.52977937 a.u.

Standard orientation:

\begin{tabular}{|c|c|c|c|c|}
\hline \multirow{2}{*}{$\begin{array}{l}\text { Center } \\
\text { Number }\end{array}$} & Atomic & \multirow{2}{*}{$\begin{array}{l}\text { Atomic } \\
\quad \text { Type }\end{array}$} & \multicolumn{2}{|c|}{ Coordinates (Angstroms) } \\
\hline & Number & & $x$ & Z \\
\hline 1 & 7 & 0.000000 & 0.000000 & 0.552563 \\
\hline 2 & 7 & 0.000000 & 0.000000 & -0.552563 \\
\hline
\end{tabular}




\subsection{Transition States Optimizations and IRC calculations}

5.2.1 Transition state for $T$ of 1 forming vinylnitrene ${ }^{32}$

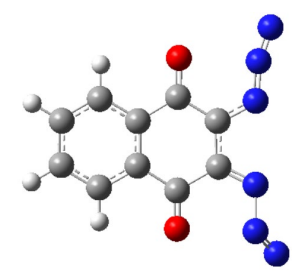

DFT/B3LYP 6-31+G(d), E = -862.26870807 a.u.

Standard orientation:

\begin{tabular}{ccccccc} 
Center & \multicolumn{2}{c}{ Atomic } & \multicolumn{2}{c}{ Atomic } & \multicolumn{3}{c}{ Coordinates (Angstroms) } \\
Number & \multicolumn{2}{c}{ Number } & Type & $X$ & $Y$ & $Z$ \\
\hline 1 & 6 & 0 & 2.682139 & -1.425418 & 0.009723 \\
2 & 6 & 0 & 1.468542 & -0.724764 & 0.022728 \\
3 & 6 & 0 & 1.473782 & 0.684846 & -0.004652 \\
4 & 6 & 0 & 2.691655 & 1.376068 & -0.047172 \\
5 & 6 & 0 & 3.893355 & 0.670330 & -0.069876 \\
6 & 6 & 0 & 3.889099 & -0.730783 & -0.042832 \\
7 & 1 & 0 & 2.657866 & -2.510029 & 0.038562 \\
8 & 1 & 0 & 2.675247 & 2.461044 & -0.061499 \\
9 & 1 & 0 & 4.835527 & 1.210214 & -0.107076 \\
10 & 1 & 0 & 4.827377 & -1.278189 & -0.060734 \\
11 & 6 & 0 & 0.208695 & 1.459862 & 0.022011 \\
12 & 8 & 0 & 0.191528 & 2.694490 & 0.044207 \\
13 & 6 & 0 & 0.198118 & -1.494259 & 0.095331 \\
14 & 8 & 0 & 0.185387 & -2.706601 & 0.234807 \\
15 & 7 & 0 & -2.257886 & 1.367689 & 0.023611 \\
16 & 6 & 0 & -1.060747 & 0.717834 & 0.018740 \\
17 & 7 & 0 & -2.337580 & 2.621595 & 0.026031 \\
18 & 7 & 0 & -2.705501 & 3.696416 & 0.028073 \\
19 & 6 & 0 & -1.108044 & -0.727311 & -0.019797 \\
20 & 7 & 0 & -2.281356 & -1.281036 & -0.159623 \\
21 & 7 & 0 & -2.291032 & -2.742896 & -0.397522 \\
22 & 7 & 0 & -2.988203 & -3.465281 & 0.201346
\end{tabular}

IRC Graph:

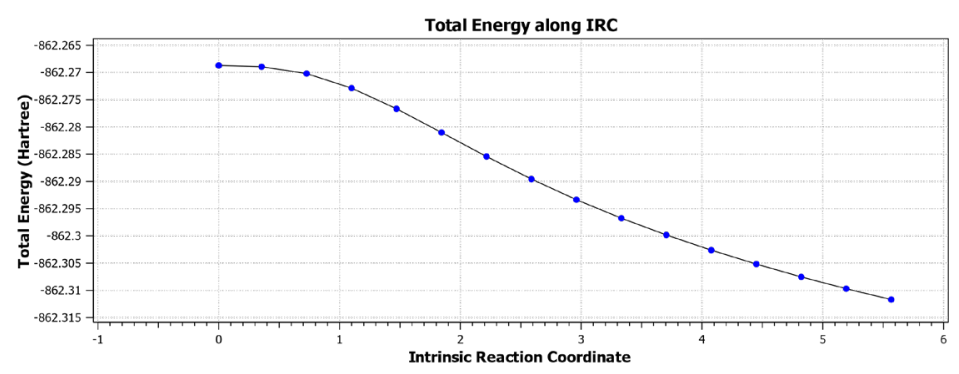


5.2.2. Transition state for vinylnitrene ${ }^{3} 2$ forming biradical ${ }^{3} 11$

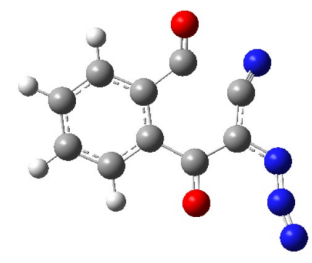

DFT/B3LYP 6-31+G(d), E = -752.76171956 a.u.

Standard orientation:

\begin{tabular}{|c|c|c|c|c|c|}
\hline \multirow{2}{*}{$\begin{array}{l}\text { Center } \\
\text { Number }\end{array}$} & Atomic & \multicolumn{2}{|c|}{ Atomic } & \multicolumn{2}{|c|}{ Coordinates (Angstrom } \\
\hline & Numbe & & Type & $x$ & Z \\
\hline 1 & 6 & 0 & 3.028177 & 0.303626 & -0.164514 \\
\hline 2 & 6 & 0 & 1.627432 & 0.411315 & -0.194783 \\
\hline 3 & 6 & 0 & 0.815471 & -0.719725 & 0.039446 \\
\hline 4 & 6 & 0 & 1.438408 & -1.949411 & 0.300482 \\
\hline 5 & 6 & 0 & 2.828092 & -2.045531 & 0.355282 \\
\hline 6 & 6 & 0 & 3.627554 & -0.919606 & 0.123119 \\
\hline 7 & 1 & 0 & 3.625190 & 1.188766 & -0.362369 \\
\hline 8 & 1 & 0 & 0.815272 & -2.823334 & 0.457075 \\
\hline 9 & 1 & 0 & 3.290901 & -3.003777 & 0.574840 \\
\hline 10 & 1 & 0 & 4.710457 & -0.998348 & 0.163887 \\
\hline 11 & 6 & 0 & -0.683118 & -0.731926 & -0.034792 \\
\hline 12 & 8 & 0 & -1.277089 & -1.799336 & -0.241003 \\
\hline 13 & 6 & 0 & 1.017183 & 1.715556 & -0.501924 \\
\hline 14 & 8 & 0 & 1.518794 & 2.724649 & -0.881143 \\
\hline 15 & 7 & 0 & -2.860148 & 0.432920 & 0.102774 \\
\hline 16 & 6 & 0 & -1.493871 & 0.469565 & 0.171480 \\
\hline 17 & 7 & 0 & -3.486416 & -0.615569 & -0.194655 \\
\hline 18 & 7 & 0 & -4.291001 & -1.380991 & -0.439332 \\
\hline 19 & 6 & 0 & -0.985548 & 1.749343 & 0.557641 \\
\hline 20 & 7 & 0 & -1.033027 & 2.782919 & 1.136230 \\
\hline
\end{tabular}

IRC Graph:

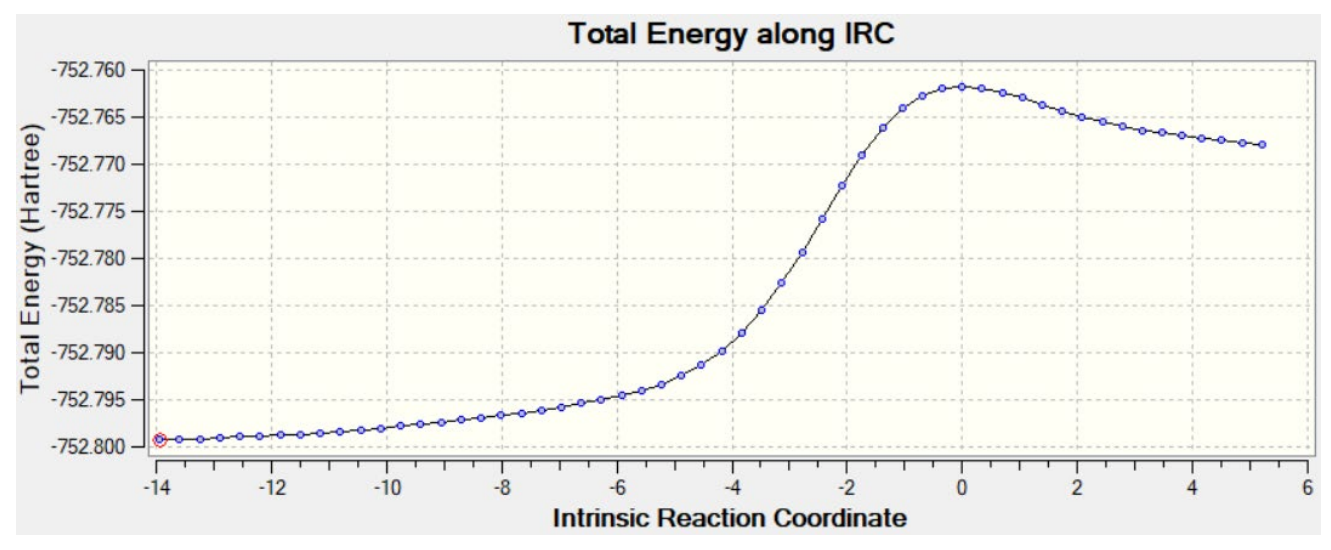


5.2.3. Transition state for biradical ${ }^{3} 11$ forming biradical ${ }^{3} 12$

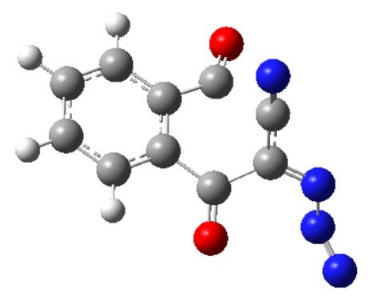

DFT/B3LYP 6-31+G(d), E = -752.75172681 a.u.

Standard orientation:

\begin{tabular}{|c|c|c|c|c|c|}
\hline \multirow{2}{*}{$\begin{array}{l}\text { Center } \\
\text { Number }\end{array}$} & \multicolumn{2}{|c|}{ Atomic } & Atomic & \multicolumn{2}{|c|}{ Coordinates (Angstro } \\
\hline & & & Type & $Y$ & Z \\
\hline 1 & 6 & 0 & -2.834924 & 0.634444 & -0.5056 \\
\hline 2 & 6 & 0 & -1.438180 & 0.495675 & -0.459435 \\
\hline 3 & 6 & 0 & -0.851688 & -0.699051 & 0.000088 \\
\hline 4 & 6 & 0 & -1.673168 & -1.767046 & 0.377173 \\
\hline 5 & 6 & 0 & -3.06 & -1.62 & 0.344072 \\
\hline 6 & 6 & 0 & -3.642047 & -0.423119 & -0.091215 \\
\hline 7 & 1 & 0 & -3.266 & 1.560255 & -0.873899 \\
\hline 8 & 1 & 0 & -1.219564 & -2.697690 & 0.703804 \\
\hline 9 & 1 & 0 & -3.694483 & -2.446988 & 0.656091 \\
\hline 10 & 1 & 0 & -4.7 & -0.32 & -0.119802 \\
\hline 11 & 6 & 0 & 0.633946 & -0.876760 & 0.041949 \\
\hline 12 & 8 & 0 & 1.1 & -1.90 & -0.343993 \\
\hline 13 & 6 & 0 & -0.549750 & 1.570325 & -0.931541 \\
\hline 14 & 8 & 0 & -0.769686 & 2.595609 & -1.486090 \\
\hline 15 & 6 & 0 & 1.502225 & 0.23 & 0.611623 \\
\hline 16 & 6 & 0 & 0.959097 & 1.095000 & 1.626774 \\
\hline 17 & 7 & 0 & 0.497912 & 1.734339 & 2.483108 \\
\hline 18 & 7 & 0 & 2.759129 & 0.402558 & 0.338187 \\
\hline 19 & 7 & 0 & 3.272375 & -0.286126 & -0.773443 \\
\hline 20 & 7 & 0 & 4.248173 & -0.921994 & -0.877672 \\
\hline
\end{tabular}

\section{IRC Graph:}

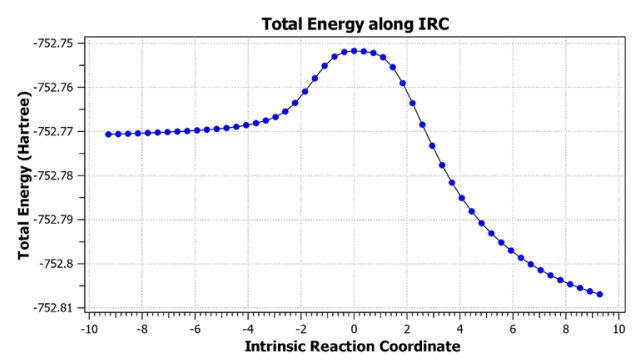


5.2.4. Transition state for biradical ${ }^{3} 12$ forming alkylnitrene ${ }^{3} 2$

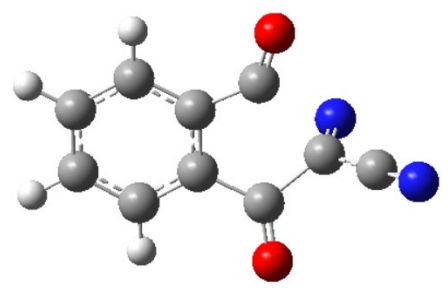

DFT/B3LYP 6-31+G(d), E = -643.281922 a.u.

Standard orientation:

\begin{tabular}{|c|c|c|c|c|}
\hline \multirow{2}{*}{$\begin{array}{l}\text { Center } \\
\text { Number }\end{array}$} & Atomic & Atomic & \multicolumn{2}{|c|}{ Coordinates (Angstroms) } \\
\hline & Numbe & Type & Y & Z \\
\hline 1 & 6 & 2.154151 & 1.340358 & -0.089217 \\
\hline 2 & 6 & 0.885538 & 0.746477 & -0.035072 \\
\hline 3 & 6 & 0.745348 & -0.652297 & -0.017399 \\
\hline 4 & 6 & 1.878684 & -1.469069 & -0.060611 \\
\hline 5 & 6 & 3.142665 & -0.877839 & -0.102320 \\
\hline 6 & 6 & 3.280792 & 0.518113 & -0.117376 \\
\hline 7 & 1 & 2.243887 & 2.422096 & -0.115109 \\
\hline 8 & 1 & 1.760021 & -2.548083 & -0.053924 \\
\hline 9 & 1 & 4.028940 & -1.505712 & -0.125140 \\
\hline 10 & 1 & 4.271497 & 0.962362 & -0.155945 \\
\hline 11 & 6 & -0.626785 & -1.223238 & 0.010853 \\
\hline 12 & 8 & -0.902472 & -2.359125 & -0.301874 \\
\hline 13 & 6 & -0.362400 & 1.518788 & -0.066853 \\
\hline 14 & 8 & -0.604232 & 2.678120 & -0.072085 \\
\hline 15 & 7 & -1.829468 & -0.101880 & 1.803325 \\
\hline 16 & 6 & -1.739044 & -0.260442 & 0.524940 \\
\hline 17 & 6 & -2.868967 & -0.027213 & -0.345438 \\
\hline 18 & 7 & -3.769189 & 0.164100 & -1.055791 \\
\hline
\end{tabular}

IRC Graph:

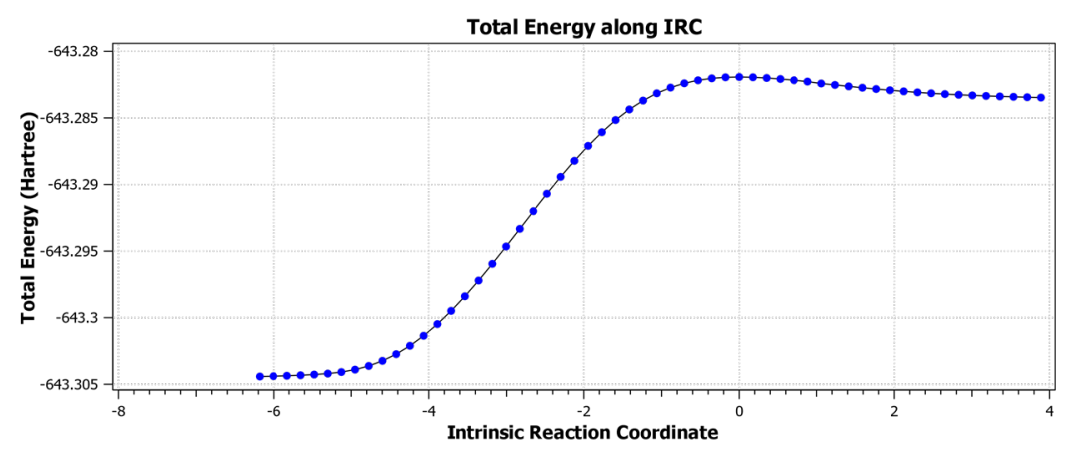


5.2.5. Transition state for vinylnitrene ${ }^{3} 2$ forming triplet diimino diradical ${ }^{3} 14$

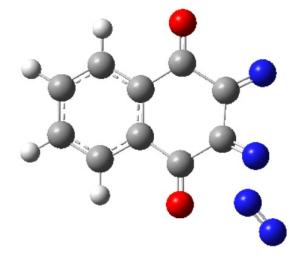

DFT/B3LYP 6-31+G(d), E = -752.77011923 a.u.

Standard orientation:

\begin{tabular}{|c|c|c|c|c|c|}
\hline \multirow{2}{*}{$\begin{array}{l}\text { Center } \\
\text { Number }\end{array}$} & \multicolumn{2}{|l|}{ Atomic } & Atomic & \multicolumn{2}{|c|}{ Coordinates (Angstroms } \\
\hline & Numbe & & Type & $Y$ & Z \\
\hline 1 & 6 & 0 & 2.898500 & 0.455836 & -0.216497 \\
\hline 2 & 6 & 0 & 1.503839 & 0.440513 & -0.073272 \\
\hline 3 & 6 & 0 & 0.832127 & -0.788730 & 0.120759 \\
\hline 4 & 6 & 0 & 1.572087 & -1.979475 & 0.173765 \\
\hline 5 & 6 & 0 & 2.957735 & -1.951714 & 0.037638 \\
\hline 6 & 6 & 0 & 3.621783 & -0.733180 & -0.159353 \\
\hline 7 & 1 & 0 & 3.394279 & 1.409486 & -0.366757 \\
\hline 8 & 1 & 0 & 1.040673 & -2.914737 & 0.317053 \\
\hline 9 & 1 & 0 & 3.523238 & -2.878245 & 0.082173 \\
\hline 10 & 1 & 0 & 4.702789 & -0.713597 & -0.265805 \\
\hline 11 & 6 & 0 & -0.648461 & -0.880871 & 0.241717 \\
\hline 12 & 8 & 0 & -1.230970 & -1.938824 & 0.416042 \\
\hline 13 & 6 & 0 & 0.779732 & 1.741047 & -0.120099 \\
\hline 14 & 8 & 0 & 1.311616 & 2.789952 & -0.420391 \\
\hline 15 & 7 & 0 & -2.688034 & 0.480930 & -0.212702 \\
\hline 16 & 6 & 0 & -1.440368 & 0.400462 & 0.082223 \\
\hline 17 & 7 & 0 & -3.337395 & -0.829632 & -0.616237 \\
\hline 18 & 7 & 0 & -4.362594 & -1.215466 & -0.242422 \\
\hline 19 & 6 & 0 & -0.707020 & 1.694202 & 0.270091 \\
\hline 20 & 7 & 0 & -1.258530 & 2.692672 & 0.803687 \\
\hline
\end{tabular}

IRC Graph:

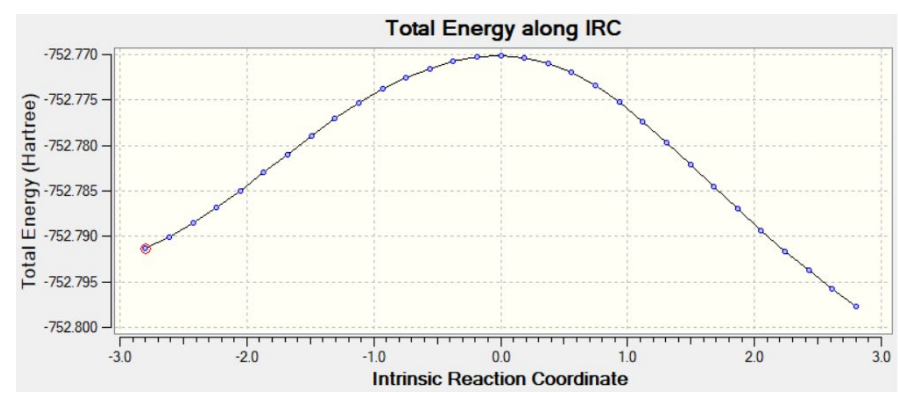


5.2.6. Transition state diimino diradical ${ }^{3} 14$ forming biradical ${ }^{3} 12$

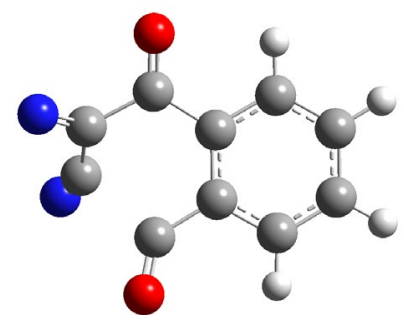

DFT/B3LYP 6-31+G(d), E = -643.271661 a.u.

Standard orientation:

Center Atomic Atomic Coordinates (Angstroms)

Number Number Type X Y Z

$\begin{array}{lllll}1 & 6 & 1.978337 & -1.353168 & -0.110868 \\ 2 & 6 & 0.730300 & -0.712596 & -0.175365 \\ 3 & 6 & 0.632296 & 0.686267 & -0.015069 \\ 4 & 6 & 1.802666 & 1.427899 & 0.195126 \\ 5 & 6 & 3.039550 & 0.788306 & 0.266205 \\ 6 & 6 & 3.129373 & -0.601154 & 0.114434 \\ 7 & 1 & 2.027765 & -2.430398 & -0.236442 \\ 8 & 1 & 1.722392 & 2.504729 & 0.301402 \\ 9 & 1 & 3.938172 & 1.373952 & 0.438450 \\ 10 & 1 & 4.095770 & -1.094825 & 0.166055 \\ 11 & 6 & -0.661415 & 1.438658 & -0.097458 \\ 12 & 8 & -0.701086 & 2.651105 & -0.123943 \\ 13 & 6 & -0.476898 & -1.526082 & -0.382143 \\ 14 & 8 & -0.626974 & -2.626693 & -0.793013 \\ 15 & 7 & -2.941574 & 1.014724 & -0.861357 \\ 16 & 6 & -1.977858 & 0.637175 & -0.132171 \\ 17 & 6 & -2.059801 & -0.582847 & 0.662661 \\ 18 & 7 & -2.483986 & -1.266652 & 1.534796 \\ ---------------------------------------------------------\end{array}$

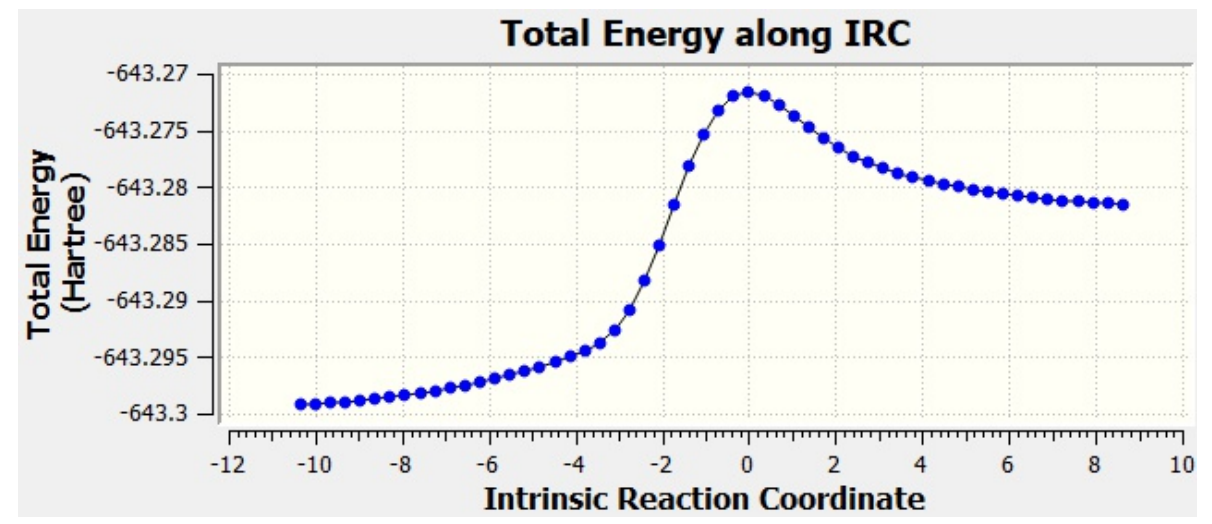




\subsection{Calculated spin densities of $1, T_{1}$ of 1 , vinylnitrene ${ }^{3} 2$, and alkylnitrene ${ }^{3} 3$.}

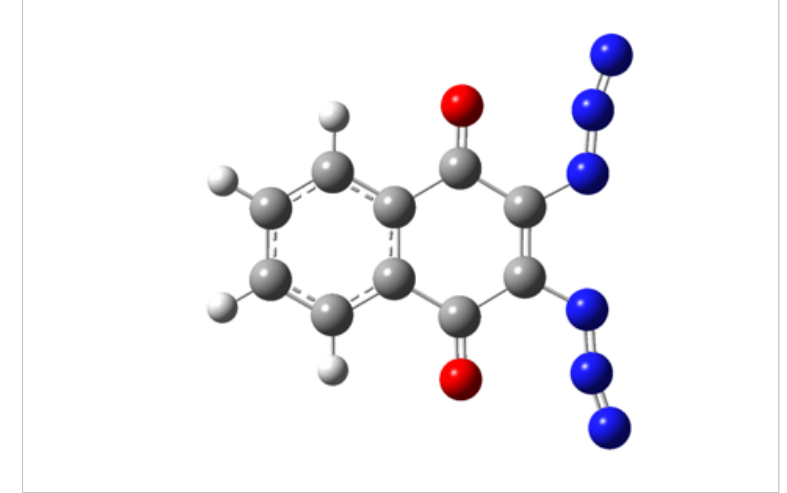

$\mathrm{S}_{0}$ of $1(0)$

$\mathrm{C}=\mathrm{C} 1.38 \AA, \mathrm{C}=\mathrm{O} 1.23 \AA$, C-N $1.38 \AA$

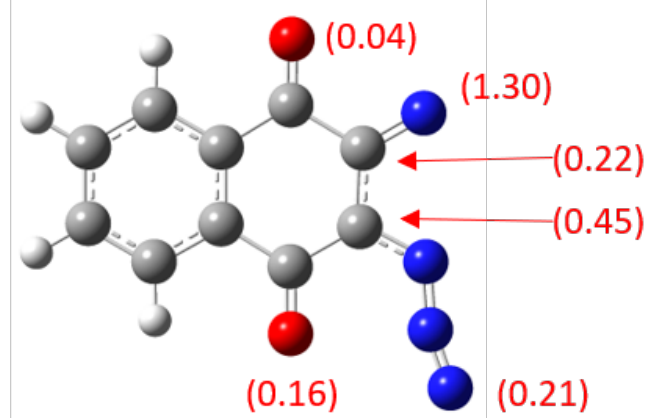

Triplet vinylnitrene $2(-2)$

$C=C 1.45 \AA, \beta-C=01.22 \AA \gamma-C=01.24 \AA$, $\alpha-C-N 1.28 \AA$

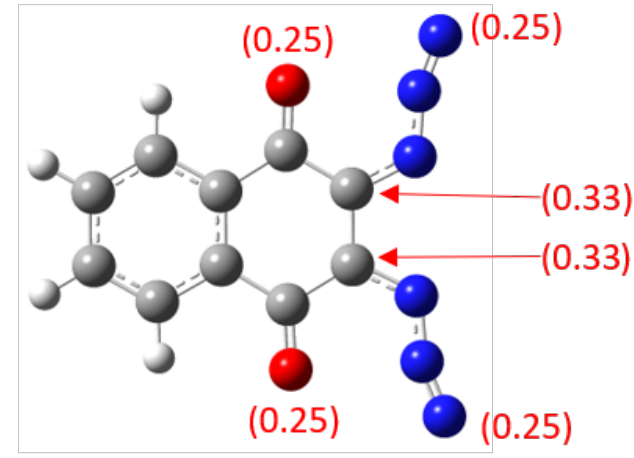

$\mathrm{T}_{1}$ of $1(30)$

$\mathrm{C}=\mathrm{C} 1.46 \AA, \mathrm{C}=\mathrm{O} 1.25 \AA, \mathrm{C}-\mathrm{N} 1.35 \AA$

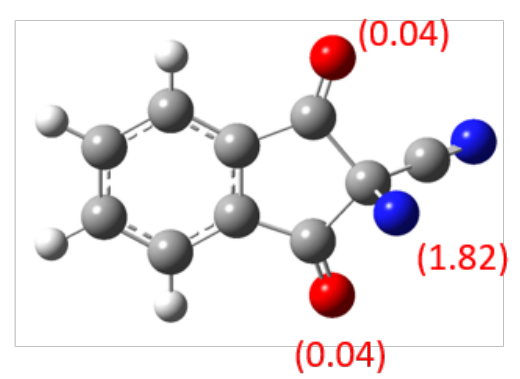

Triplet alkylnitrene 3 (-24) $\mathrm{C}=\mathrm{O} 1.20 \AA, \beta-\mathrm{C}-\mathrm{N} 1.42 \AA$

Figure S11. Optimized structures of $\mathbf{1}, \mathrm{T}_{1}$ of $\mathbf{1},{ }^{\mathbf{3}} \mathbf{2}$, and ${ }^{3} \mathbf{3}$.

\section{References}

1. Frisch, M. J.; Trucks, G. W.; Schlegel, H. B.; Scuseria, G. E.; Robb, M. A.; Cheeseman, J. R.; Scalmani, G.; Barone, V.; Mennucci, B.; Petersson, G. A.; Nakatsuji, H.; Caricato, M.; Li, X.; Hratchian, H. P.; Izmaylov, A. F.; Bloino, J.; Zheng, G.; Sonnenberg, J. L.; Hada, M.; Ehara, M.; Toyota, K.; Fukuda, R.; Hasegawa, J.; Ishida, M.; Nakajima, T.; Honda, Y.; Kitao, O.; Nakai, H.; Vreven, T.; Montgomery, J. A.; Peralta, J. E.; Ogliaro, F.; Bearpark, M.; Heyd, J. J.; Brothers, E.; Kudin, K. N.; Staroverov, V. N.; Kobayashi, R.; Normand, J.; Raghavachari, K.; Rendell, A.; Burant, J. C.; Iyengar, S. S.; Tomasi, J.; Cossi, M.; Rega, N.; Millam, J. M.; Klene, M.; Knox, J. E.; Cross, J. B.; Bakken, V.; Adamo, C.; Jaramillo, J.; Gomperts, R.; Stratmann, R. E.; Yazyev, O.; Austin, A. J.; Cammi, R.; Pomelli, C.; Ochterski, J. W.; Martin, R. L.; Morokuma, K.; Zakrzewski, V. G.; Voth, G. A.; Salvador, P.; Dannenberg, J. J.; Dapprich, S.; Daniels, A. D.; Farkas; Foresman, J. B.; Ortiz, J. V.; Cioslowski, J.; Fox, D. J., Gaussian 09, Revision B.01. Wallingford CT, 2009.

2. Becke, A. D., Density-functional thermochemistry. III. The role of exact exchange. J. Chem. Phys. 1993, 98 (7), 5648-5652.

3. Lee, C.; Yang, W.; Parr, R. G., Development of the Colle-Salvetti correlation-energy formula into a functional of the electron density. Phys. Rev. B 1988, 37 (2), 785-789. 
4. Bauernschmitt, R.; Ahlrichs, R., Treatment of electronic excitations within the adiabatic approximation of time dependent density functional theory. Chem. Phys. Lett. 1996, 256 (4), 454464.

5. Foresman, J. B.; Head-Gordon, M.; Pople, J. A.; Frisch, M. J., Toward a systematic molecular orbital theory for excited states. J. Phys. Chem. 1992, 96 (1), 135-149.

6. Stratmann, R. E.; Scuseria, G. E.; Frisch, M. J., An efficient implementation of time-dependent density-functional theory for the calculation of excitation energies of large molecules. J. Chem. Phys. 1998, 109 (19), 8218-8224.

7. Gonzalez, C.; Schlegel, H. B., An improved algorithm for reaction path following. J. Chem. Phys. 1989, 90 (4), 2154-2161.

8. Gonzalez, C.; Schlegel, H. B., Reaction path following in mass-weighted internal coordinates. J. Phys. Chem. 1990, 94 (14), 5523-5527.

9. Ochwat, F., Chem. Ber. 1923, 56, 1298. 